\title{
Del sedentarismo a la hipermovilidad. Medida y determinantes de las historias de (in)movilidad residencial en contextos urbanos*
}

\author{
Isabel Palomares-Linares \\ Universidad de Granada, Departamento de Sociología \\ ipalomares@ugr.es (autor para correspondencia)

\section{Maarten van Ham} \\ Delft University of Technology, OTB - Research for the Built Environment. \\ Faculty of Architecture and the Built Environment. Delft, The Netherlands \\ University of St Andrews. School of Geography and Sustainable Development. \\ Scotland, UK \\ m.vanham@tudelft.nl
}

\section{Resumen}

A pesar del bagaje acumulado en otros países, en España, las fuentes de microdatos disponibles en materia de movilidad han resultado insuficientes para afrontar análisis de las historias individuales residenciales y espaciales dentro de contextos urbanos. Este trabajo permite realizar una primera aproximación al estudio de dichas historias en un contexto metropolitano español. En base a una encuesta de Granada con información acerca del total de cambios domiciliarios y espaciales —entre barrios- realizados en un periodo de diez años, proponemos un método de regresión de conteo Hurdle para el estudio de las historias de (in)movilidad y descubrimos la influencia de cuatro dimensiones básicas (transcurso vital, estado en la carrera residencial, posición social y tipo de barrio en el que se reside) en la generación de trayectorias más (o menos) sedentarias. Los resultados de este artículo indican que la inmovilidad residencial y espacial responde a factores como el grado de arraigo de los individuos y de los hogares. La hipermovilidad, sin embargo, está conectada con perfiles jóvenes y con pocas cargas familiares. La posición social y el tipo de barrio en el que se reside emergen como factores explicativos relevantes a la hora de entender trayectorias más sedentarias.

Palabras clave: (in)movilidad residencial; (in)movilidad espacial; regresión de conteo; transcurso vital; carrera residencial; posición social; barrio

* Este artículo muestra resultados enmarcados en el proyecto de investigación titulado Procesos de reconfiguración social metropolitana, código: CSO2014-55780-C3-3-P. 
Abstract. From Immobility to Hypermobility: Measure and Determinants of (im)Mobility Trajectories in Urban Contexts

Although there is a large international body of literature on residential mobility, the research on Spain is scarce. The main reason is that there is little suitable microdata which allows the analysis of individual residential and spatial mobility within urban contexts. This paper is one of the first to study mobility in a Spanish metropolitan context. We use data from a survey conducted in Granada with information on residential and spatial mobility between neighborhoods over a 10-year period and a Hurdle count regression method for the study of (in)mobility. The results show the effects of four basic dimensions (life course, stage in the residential career, social position and the neighborhoods where people live) on the generation of more (or less) sedentary trajectories. We found that residential and spatial immobility is associated with settled individuals and households. On the other hand, hypermobility is connected to younger age groups and those without children. The social position and type of neighborhood in which people reside emerge as relevant factors to understand immobility behavior.

Keywords: residential (im)mobility; spatial (im)mobility; count regression; life course; housing career; socioeconomic status; neighborhood

\author{
Sumario \\ 1. Introducción 5. Discusión y conclusiones \\ 2. Estado de la cuestión Referencias bibliográficas \\ 3. Metodología Anexos \\ 4. Resultados
}

\title{
1. Introducción
}

La movilidad residencial, entendida como todo cambio de domicilio que se origina y que finaliza dentro un mismo entorno de vida (Courgeau, 1988) ha sido fruto de una creciente atención durante las últimas décadas. El interés académico y político que despiertan otros movimientos — como las migraciones internacionales - es mayor, pero una trayectoria cada vez más amplia de estudios específicos ha demostrado que los perfiles y los actores -individuales y colectivos- que participan en las migraciones de corta y larga distancia difieren, así como el marco de oportunidades y de constricciones implicados en las decisiones (Clark, 1982; Nivalainen, 2004). Las personas cambian de domicilio motivadas por contextos y por razones diferentes a las que actúan en la elección de migrar.

Aunque los primeros trabajos se centraron en explicar las motivaciones implicadas en las decisiones de los cambios (Chevan, 1971; Goodman, 1976), en la actualidad, observamos un renovado interés en descubrir los factores detrás de las decisiones de permanencia (Fischer y Malmberg, 2001; Clark et al., 2015). Más aún, nuevos planteamientos conceptuales señalan la necesidad de entender la (in)movilidad residencial como un proceso relacional que une (o en ocasiones ata) a las personas con los espacios a lo largo de la vida (Coulter et 
al., 2016). Desde esta perspectiva, orientada a explicar el comportamiento residencial más que las decisiones puntuales, movilidad e inmovilidad no son antónimos, sino fenómenos interconectados que componen historias de vida más o menos sedentarias, pero también se trata de fenómenos interconectados con el contexto donde se desarrollan (Thomas et al., 2015). El espacio, entendido aquí como lugar social y físico, no forma parte del escenario donde tienen lugar las historias, sino de la acción. En este sentido, las trayectorias de (in)movilidad no solo se refieren a los cambios de domicilio, sino también a los cambios y a las permanencias en el espacio. Las personas pueden desarrollar historias más o menos móviles en términos domiciliarios, pero permaneciendo en el mismo lugar o trazando un recorrido más amplio dentro del contexto urbano (Clark et al., 2006). El barrio, como entorno residencial más cercano (Kemeny, 1991) y por sus implicaciones en la vida social de sus habitantes (Kleinhans, 2009; De Pablos y Susino, 2010), es una unidad fundamental de este análisis espacial.

El presente estudio parte de esta definición más amplia y supone un intento por ahondar en la explicación de las historias de (in)movilidad en sus dimensiones residenciales y espaciales. En países donde se dispone de amplios registros longitudinales se han realizado múltiples aportaciones al respecto (Stovel y Bolan, 2004; Coulter y van Ham, 2013; van Ham et al., 2014). En España, la dificultad de contar con fuentes de datos para construir y estudiar historias completas de (in)movilidad ha impedido la adopción de esta perspectiva. Por ello, y contando con información retrospectiva única sobre movilidad en el área metropolitana de Granada, la primera cuestión que guía la investigación es cómo se pueden estudiar las historias de (in)movilidad residencial y espacial con los datos disponibles. Esta cuestión requiere de una respuesta metodológicamente fundada, pero también plantea resultados interesantes en el plano conceptual, dado que, por primera vez en el contexto español, estudiamos no solo la ocurrencia de (in)movilidad residencial y espacial, sino también la intensidad con la que se produce.

Una vez ajustado un modelo de análisis adecuado y consistente con la realidad a estudiar, la segunda cuestión de este artículo, de calado más explicativo, pasa por conocer qué factores están actuando en la generación de un comportamiento más o menos sedentario en términos residenciales y espaciales. A este respecto, y aun cuando la experiencia de estudios sobre movilidad y sedentarismo urbano señala la influencia de una pluralidad de factores, nosotros analizamos indicadores de cuatro dimensiones advertidas y aceptadas como básicas en la explicación de la (in)movilidad: el transcurso vital (Elder, 1985; Mulder y Hooimeijer, 1999); el estado en la carrera residencial - housing career - (Kendig, 1984; Clark et al., 2003); la posición social de hogares e individuos (Clark y Dieleman, 1996; Crowder y South, 2005), y aspectos socioespaciales como las características y el tipo de barrio en el que se reside (Clark et al., 2006; Rabe y Taylor, 2010) o los lazos y las ataduras sociales acumulados en el contexto inmediato del barrio (Dawkins, 2006; Kan, 2007). Contestando a esta segunda cuestión, avanzamos en el conocimiento de la influencia que estos factores están ejerciendo en conductas residenciales que van desde el sedentarismo hasta la hipermovilidad urbana. 


\section{Estado de la cuestión}

\subsection{Más allá de la decisión de moverse o quedarse. Una apuesta para el estudio de la (in)movilidad en contextos urbanos}

Desde una perspectiva micro, el estudio de la movilidad residencial ha estado más enfocado en explicar por qué las personas deciden mudarse a lo largo de sus vidas (Sabagh et al., 1969; Chevan, 1971; Dieleman, 2001; Módenes y Cabré, 2002). Bajo este prisma centrado en las decisiones de movilidad, la explicación del sedentarismo o de la inmovilidad ha quedado relegada a un segundo plano, entendida en muchas ocasiones como un estado de latencia entre movimientos o como una acción no estratégica, sino reactiva o táctica. Poniendo el foco en los motivantes de los cambios, la inmovilidad se ha relacionado con la falta de necesidades de vivienda (Goodman, 1976; Clark y Dieleman, 1996) o con las dificultades para satisfacerlas (Rossi, 1955; Kan, 1999).

A pesar del dominio del paradigma de la movilidad, hoy en día, fenómenos como el descenso de las tasas de movilidad registrado en países con mayor dinamismo residencial (Cooke, 2011) o el impacto de la crisis económica en los sistemas y en las carreras residenciales (Palomares-Linares y van Ham, 2016) han fomentado un renovado interés en comprender el sedentarismo y sus causas. A la famosa cuestión que daba título a la pionera obra de Rossi (1955), "¿por qué se mueven las familias?», se le suman ahora interrogantes como ¿por qué se quedan? (Meeus y De Decker, 2015) o ¿por qué lo hacen durante más o menos tiempo? (Hjälm, 2014; Morrison y Clark, 2016). Desde una perspectiva basada en la toma de decisiones, estos acercamientos señalan que la inmovilidad es algo más que el reverso de la movilidad y advierten de la necesidad de ampliar el marco conceptual y analítico en los estudios sobre decisiones residenciales, ya que las razones y las dinámicas que impulsan a moverse pueden no ser las mismas que las que impulsan a permanecer en un lugar (Clark y Lisowski, 2017).

Aun cuando estos planteamientos avanzan en el conocimiento redirigiendo la atención sobre el sedentarismo, la explicación del comportamiento residencial no se agota abarcando las respuestas posibles en una misma decisión. Desde una perspectiva longitudinal, recientes estudios apuestan por una visión relacional entre movilidad e inmovilidad, en la que la cuestión a comprender no son las elecciones puntuales, sino las trayectorias de (in)movilidad (Stovel y Bolan, 2004; Coulter et al., 2016). Desde esta visión, más centrada en explicar comportamientos más amplios —enlazando las acciones y las elecciones concretas-, los cambios y las permanencias son capítulos de una misma historia residencial. Coulter y van Ham (2013), en un estudio sobre historias individuales de (in)movilidad, comprueban que las trayectorias residenciales se caracterizan por tener una cierta inercia a lo largo del tiempo. Hay grupos de personas consistentemente sedentarios o permanentemente móviles. Otros estudios demuestran, a su vez, la inercia que las decisiones y las trayectorias de (in)movilidad pasadas tienen en las decisiones presentes (Fischer y Malmberg, 2001) o en las expectativas futuras (Thomas et al., 2016). 
Figura 1. Propuesta para el estudio de la (in)movilidad en contextos urbanos

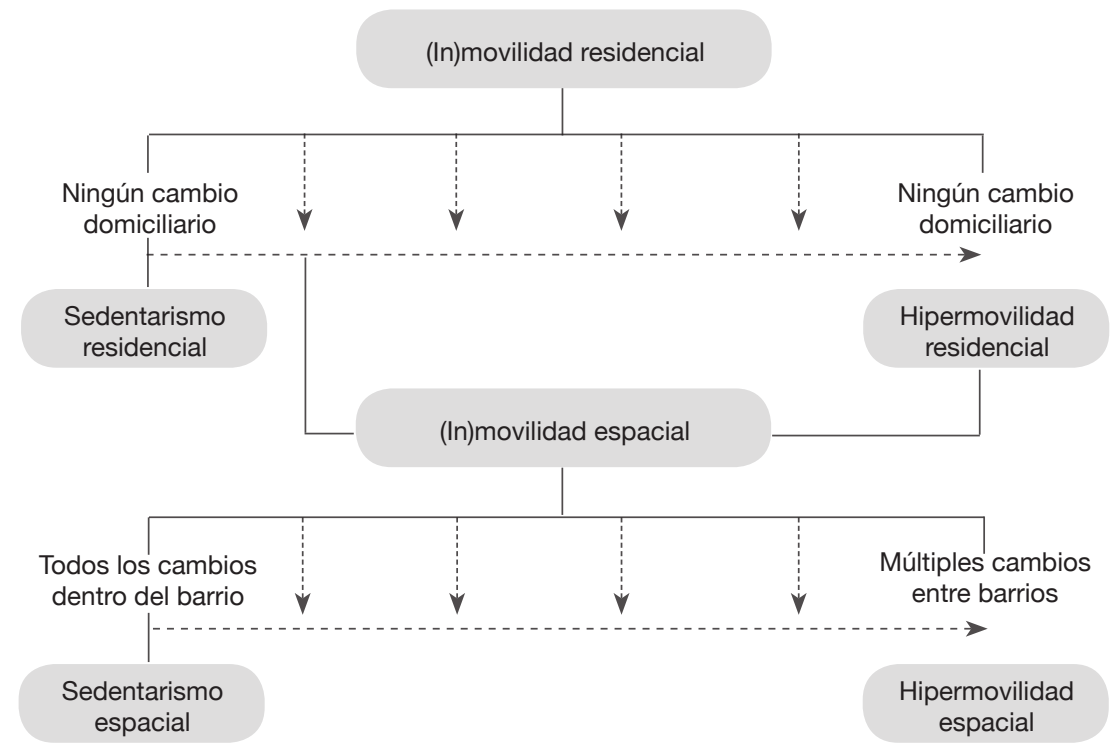

Fuente: elaboración propia.

Pero el comportamiento residencial tiene una dimensión espacial más allá de la meramente domiciliaria. Kemeny (1991), al hablar de residencia, apela a un concepto amplio en el que se entrelazan distintos niveles. La vivienda es el nivel básico y privado en el que se desarrolla la residencia, pero el barrio, como entorno inmediato donde se desarrolla parte de la vida diaria, conforma un segundo nivel. En este sentido, las personas pueden moverse más o menos, pero permanecer en el mismo lugar de vida concreto - el barrio- o trazar trayectorias más amplias a lo largo del espacio urbano (Clark et al., 2006; Susino y Palomares-Linares, 2013). Por ello, en la explicación del comportamiento residencial, el estudio de las trayectorias de (in)movilidad estrictamente domiciliaria debe completarse procurando un mejor entendimiento de la (in) movilidad espacial de dichas trayectorias. En la figura 1 y como resumen de las ideas plasmadas en este apartado, presentamos nuestra propuesta para el estudio de las trayectorias de (in)movilidad.

\subsection{Dimensiones básicas en la explicación de (in)movilidad residencial y espacial}

Los primeros estudios enfocados al análisis de la movilidad individual descubrieron la profunda relación existente entre ciclos familiares, necesidades de vivienda-hogar y movilidad (Leslie y Richardson, 1961; Chevan, 1971; Goodman, 1976), o la interconexión entre las decisiones residenciales y los 
lugares implicados en las elecciones (Sabagh et al., 1969). Hoy en día, y tras una amplia experiencia de investigaciones cada vez más específicas, realizar una compilación de los factores implicados en las decisiones y en las trayectorias de movilidad e inmovilidad residencial y espacial resultaría una ardua tarea. Se han señalado desde factores estructurales, como rasgos culturales o características de los sistemas de vivienda (Long, 1991; Meeus y De Decker, 2015), hasta factores individuales, como las percepciones y las emociones que generan los cambios (Oishi y Talhelm, 2012) o las valoraciones que las personas realizan de su entorno residencial (Thomas et al., 2015). Efectivamente, este bagaje apunta la inmensa complejidad con la que nos encontramos a la hora de entender los comportamientos residenciales. Sin embargo, en este artículo, abordamos las cuatro dimensiones básicas que, bajo nuestro punto de vista - y fundamentados en la revisión bibliográfica-, explican la (in)movilidad en términos residenciales y espaciales: el transcurso vital; el estado en la carrera residencial; la posición social, y el tipo de barrio y los lazos sociales generados en el mismo.

\section{El transcurso vital}

La relevancia del curso vital en la (in)movilidad es poco cuestionable. De hecho, reconocer la intersección del transcurso vital con el transcurso residencial supone la adopción de una perspectiva conceptual a partir de la que enfrentar diferentes análisis (Elder, 1985; Mulder y Hooimeijer, 1999). En contraposición con los primeros trabajos, en los que se anteponían los periodos y los estados en el ciclo vital (Rossi, 1955), la perspectiva del curso vital pone el acento en el proceso de acontecimientos y en cómo la experiencia de vida se conecta con la experiencia residencial en el corto y en el largo plazo (Elder, 1985). Así, acontecimientos como la emancipación y los primeros años de autonomía fuera del hogar parental (Mulder y Manting, 1994), el nacimiento de hijos y el crecimiento de los hogares (Michielin y Mulder, 2008), las rupturas conyugales (Clark y Lisowski, 2017) o la aparición de situaciones de dependencia funcional (Smits, 2010) funcionan como desencadenantes de movilidad. Sin embargo, cuando la calidad de los datos imposibilita la disposición de los eventos vitales, hay dos factores que han servido ampliamente como indicadores de la influencia del curso vital en la (in)movilidad: la edad y la configuración del hogar.

Dado que los acontecimientos con mayor peso de cara a producir movimientos suelen concentrarse durante las etapas de juventud y tránsito a la adultez (emancipación, inicio de la convivencia en pareja, crecimiento familiar), existe una relación lineal negativa entre la edad y la probabilidad de cambiar de domicilio que solo varía levemente durante las últimas etapas de la vida, en las que los fenómenos que afectan a la autonomía personal pueden conducir a una mayor movilidad (Elder, 1975; Clark, 2013). Por otra parte, a más tiempo vivido en un lugar, mayores lazos se forjan con el entorno (Fischer y Malmberg, 2001), a causa de ello, no solo es más probable que los jóvenes se muevan, sino que lo hagan trazando recorridos más amplios que cohortes adultas más consolidadas en términos domiciliarios y espaciales. El mismo proceso se ha 
relacionado con el comportamiento más sedentario de las familias respecto a hogares conformados por parejas o por personas solas, ya que estos hogares adquieren mayores y más diversos compromisos espaciales (por ejemplo: la escolarización) (DaVanzo, 1981; Clark y Dieleman, 1996). Por último, puesto que la hipermovilidad es un patrón asociado a los nuevos imaginarios culturales y a las estructuras socioeconómicas de la modernidad avanzada (Aramburu, 2015), las generaciones jóvenes, así como los hogares con menores ataduras, serán más propensas a adoptar historias más móviles que las generaciones y los hogares consolidados, cuyos imaginarios o ideales residenciales corresponden a otro contexto sociotemporal, el dominante en España durante la segunda mitad del siglo XX y que se caracteriza por la escasa incidencia de la movilidad residencial una vez realizados los primeros movimientos de emancipación (Módenes y López-Colás, 2014).

\section{El estado en la carrera residencial}

El término housing career, que hemos traducido como carrera residencial, pone de relieve que, en paralelo a los cursos familiares y a las carreras laborales, la movilidad también responde a una estrategia en términos de vivienda (Kendig, 1984). Tal como Clark et al. (2003: 145) formulan, la idea que hay detrás del concepto de carrera residencial es que "los hogares, en cada movimiento, buscan acercarse a la vivienda que mejor se ajuste a sus necesidades y a sus aspiraciones residenciales". Por ello, el estado en el que se encuentran los individuos y los hogares con respecto a sus aspiraciones y necesidades ejerce una gran influencia en las decisiones de (in)movilidad que realizan.

En este sentido, la calidad de la vivienda (Clark y Dieleman, 1996) o la satisfacción con su lugar de ubicación (Winstanley et al., 2002) constituyen factores clave, pero el indicador más claramente relacionado con las decisiones de (in)movilidad es el régimen de tenencia (Speare, 1970; Dieleman y Everaers, 1994; Helderman et al., 2004; Mulder, 2006). En los países donde el alquiler tiene una mayor presencia, sobre todo en los primeros pasos de la carrera residencial, la entrada a la propiedad ha sido considerada como uno de los pasos finales y que marcaba el inicio de periodos de sedentarismo más amplios (Kendig, 1984; Dieleman y Everaers, 1994). En España, una prolongada actuación política y un sistema inmobiliario orientados hacia la adquisición de vivienda han fomentado una cultura de la propiedad (Leal, 2010) a la que se ha llegado, en muchos casos, con el primer o los primeros movimientos tras la emancipación (Holdsworth e Irazoqui, 2002). En todo caso, tanto en España como en otros contextos, disponer de la vivienda en propiedad está intensamente relacionado con el sedentarismo. Los propietarios, ya sea por las inversiones realizadas (Quigley, 2002), ya sea por los mayores costes materiales y no materiales de un cambio de domicilio (Weinberg et al., 1981; Hiscock et al., 2001), tienden a estar más arraigados que las personas que se encuentran en régimen de alquiler. A su vez, el alquiler se ha relacionado con un estilo de vida más dinámico en cuanto a trayectorias laborales y ligero en cuanto a cargas familiares (Courgeau y Lelièvre, 1992), por lo que esperamos que las 
personas que no poseen ninguna vivienda desarrollen historias más móviles que los propietarios, tengan o no pagos hipotecarios pendientes.

\section{La posición social}

Entre la formación de deseos residenciales, la generación de expectativas de movilidad y la realización real de las intenciones iniciales, media la capacidad de los hogares para satisfacer dicha expectativa (Kan, 1999). En este sentido, la posición social es un indicador de la capacidad de poder de los distintos grupos en términos de recursos materiales, pero también instrumentales e incluso educativos o emocionales (Campbell et al., 1986). La mayor flexibilidad laboral y la movilidad social presente en sectores profesionales (Warnes, 1986; Fors y Lennartsson, 2008) o un mayor poder adquisitivo y un conocimiento del sistema inmobiliario o financiero necesario para enfrentar cambios residenciales (Weinberg et al., 1981) son factores por los que las clases medias y acomodadas tienen una mayor probabilidad de cumplir expectativas de movilidad, así como de elegir entornos concretos donde mudarse. Por el contrario, las clases más vulnerables tienden a desarrollar historias más sedentarias. Con menores recursos y más dependientes de las redes informales localizadas (Campbell et al., 1986; Kleinhans, 2009), tienen una tendencia más marcada hacia la inmovilidad, sobre todo hacia la inmovilidad espacial (Dawkins, 2006).

En España, al contrario de lo que ocurre en estos otros países, no se han advertido grandes diferencias sociales en las decisiones de movilidad. Estudios basados en el análisis de la (in)movilidad residencial de los últimos censos indican que, aun cuando pueden existir diferencias en la calidad de las viviendas o en las direcciones espaciales de los cambios, las clases sociales tienen una probabilidad similar de movilidad y/o de permanencia (Palomares-Linares y van Ham, 2016). En un contexto cultural proclive a la propiedad como primer paso en la carrera residencial y, por tanto, más sedentaria en su comportamiento posterior, es plausible asumir que los acontecimientos relacionados con el transcurso vital cobran una mayor relevancia como factores de movilidad. Sin embargo, esta aparente igualdad social también es resultado de la forma en que se recoge información sobre movilidad en las principales fuentes de datos españolas. Teniendo informaciones que hagan referencia al total de cambios realizados, así como los barrios donde ocurren, nuestra hipótesis es que las diferencias sociales emergerán como factores significativamente asociados a la configuración de historias de (in)movilidad residencial y espacial.

\section{Barrio y lazos sociales localizados en el entorno inmediato}

En el estudio de la (in)movilidad en contextos urbanos, el barrio es una unidad residencial básica. Ninguna decisión residencial se produce ajena al contexto inmediato donde se produce (Lee et al., 1994; van Ham y Clark, 2009). Por ello, las características del barrio y las relaciones de arraigo que establecemos en el mismo son factores con influencia en las decisiones de sedentarismo y cambio. Aun cuando se sigue debatiendo sobre los posibles efectos del barrio en múltiples facetas de la vida, existe consenso en reconocer que, de existir una 
influencia, esta está basada en el carácter social de dicho entorno. Estudios realizados en distintos países europeos han puesto de relieve la importancia del tipo de barrio donde las personas han residido a lo largo de sus vidas a la hora de predecir diferencias en el comportamiento residencial de sus habitantes a corto plazo (Kleinhans, 2009) y a largo plazo (van Ham et al., 2014). Residir en áreas de relegación se ha relacionado con un mayor sedentarismo espacial. Salir del barrio resulta más difícil cuando se vive en zonas vulnerables o deprimidas (van Ham y Clark, 2009). En España, a nivel agregado, existe una amplia literatura referida al estudio de los flujos residenciales entre zonas urbanas (Módenes y Cabré, 2002; Susino y Duque-Calvache, 2013). Esta literatura señala tendencias similares a las advertidas en otros contextos. Pero, de nuevo, las limitaciones en las fuentes de datos han dificultado la realización de análisis individuales incluyendo unidades inframunicipales de cara a conocer el alcance del «efecto barrio» en las decisiones residenciales. Disponiendo de dicha información y teniendo en cuenta los resultados de estudios anteriores, creemos que el tipo de barrio (más o menos acomodado) ejercerá una influencia en las historias de (in)movilidad, aunque su peso explicativo aumente de cara a entender el sedentarismo y la movilidad espacial.

Por otra parte, no solo el tipo de barrio, sino también el grado de arraigo en el mismo, puede influir en las decisiones (Mulder y Cooke, 2009). Las personas más arraigadas tienden a variar menos su domicilio y su entorno residencial, ya que los costes psicológicos, sociales y materiales de hacerlo aumentan cuanto mayores y más diversos son los vínculos (Fischer y Malmberg, 2001; Dawkins, 2006). En este sentido, la presencia de redes sociales y familiares en el barrio desempeña un papel esencial. Tal como DaVanzo (1981) apuntó, cuanto mayor sea el capital específico localizado (location specific capital) que las personas acumulan en un determinado lugar, menos probable es que decidan moverse y, si lo hacen, la existencia de redes sociales hace más probable que el cambio se produzca en el mismo entorno (Dawkins, 2006; Clark et al., 2015). En este artículo, y disponiendo de información sobre redes sociales y familiares en el barrio de residencia, podemos acercarnos a conocer, tentativamente, qué papel desempeñan estas redes en la generación de historias más o menos móviles. En un contexto como el español, en el que la familia y las redes informales ejercen un influyente rol en distintas facetas de la vida de sus miembros (De Pablos y Susino, 2010), creemos que la presencia de dichas redes estará asociada a un mayor sedentarismo residencial, pero también a una mayor propensión a moverse permaneciendo en el mismo barrio (sedentarismo espacial).

\section{Metodología}

\subsection{Fuente de datos y selección de la muestra}

Los datos en los que se basa este trabajo provienen de la Encuesta de Población y Vivienda realizada por el Instituto de Desarrollo Regional de la Universidad de Granada y encargada por el Ayuntamiento de la ciudad en el año 2008. 
Realizada para prever las demandas de vivienda futura en el área metropolitana, la encuesta es una base de datos única para el estudio de cuestiones residenciales en el contexto español. Dada la cantidad y el detalle de interrogantes que se plantean, disponemos de información relativa a trayectorias de movilidad, convivencia y vida cotidiana en el barrio, expectativas de movilidad futura, etc. En lo que respecta a los objetivos de este trabajo, la encuesta proporciona información relativa a cada uno de los cambios de domicilio realizados en los últimos diez años, así como el ámbito espacial en el que se ha producido, lo que nos permite hacer una composición retrospectiva de las historias de (in) movilidad recientes. La muestra final, representativa de la población del área metropolitana de Granada, fue de 2.363 individuos, todos ellos mayores de 18 años y distribuidos en 77 secciones de Granada capital y 38 secciones censales de los municipios de la corona metropolitana.

De los 2.363 individuos, descartamos a las personas que no vivían en el área metropolitana al inicio del periodo analizado (diez años atrás), esto es, a los inmigrantes, tanto si procedían de otros lugares de España como del extranjero. Esta restricción es necesaria para asegurar que la población analizada haya tenido las mismas probabilidades de (in)movilidad. Dado que los inmigrantes no han residido todo el periodo en el área metropolitana (cada uno tiene su propia fecha de llegada), la probabilidad de realizar cambios residenciales y/o espaciales una vez que viven en Granada está sesgada por su menor tiempo de exposición al fenómeno. Por otro lado, y dado que las pautas de asentamiento y relocalización de inmigrantes, sobre todo los provenientes del extranjero, difieren de las pautas de los residentes habituales (Bayona i Carrasco y López Gay, 2011), incluir las trayectorias de (in)movilidad de dichos grupos provocaría sesgos explicativos, al estudiar conjuntamente procesos que posiblemente obedezcan a distintas lógicas. Una vez descartamos dicha población, la muestra total de este estudio está compuesta por 2.011 individuos, todos ellos residentes en el área metropolitana al inicio del periodo analizado (1998-2008).

\subsection{Variables en el estudio}

Las variables dependientes en este estudio son dos variables de conteo que expresan la ocurrencia y la intensidad de movilidad durante el periodo analizado. La primera variable, referida a las historias de (in)movilidad residencial, está construida como el número de cambios de domicilio metropolitanos ocurridos durante los diez años anteriores al momento de la encuesta (1998-2008). Esto es, representa la suma total de movimientos que los entrevistados han realizado dentro del área metropolitana (mismo barrio, otro barrio, otros municipios metropolitanos). La variable resultante tiene una distribución que comprende desde 0 cambios (sedentarismo residencial absoluto) hasta un máximo de 6 movimientos. La segunda variable de conteo se refiere a las historias espaciales de los movimientos y está construida como el número de cambios de barrio realizados por los móviles durante los diez años anteriores al momento de la encuesta (1998-2008). La variable resultante refleja las veces que los entrevistados han 
traspasado la frontera del barrio en sus trayectorias de movilidad y tiene una distribución desde 0 cambios (siempre se han movido dentro del mismo barrio —sedentarismo espacial absoluto-) hasta un máximo de 5 cambios ocurridos entre barrios. Dado que el análisis de la (in)movilidad espacial solo se refiere a los móviles, el total incluido en el estudio de esta segunda variable es de 885 personas (ver la tabla 1 para una descripción de las variables).

En cuanto a las variables independientes incluidas en los modelos de análisis, son factores que sirven de aproximación a las dimensiones apuntadas en el marco teórico y que han sido ampliamente utilizados en el análisis de la movilidad. La unidad de análisis es el individuo, por lo que las variables corresponden a características de la persona entrevistada, a excepción del grupo de jóvenes que vivía con sus padres diez años atrás (comienzo del periodo en el que se pregunta por la trayectoria residencial) y que aún vive en el hogar familiar. La movilidad registrada de los no emancipados durante el periodo de estudio no corresponde con decisiones propias, sino con las decisiones del hogar donde residen. En estos casos $(n=234)$, se ha utilizado la información de la persona de referencia para asegurar que las historias de (in)movilidad que pertenecen más probablemente a los padres no se analicen en base a las características de sus hijos.

La edad y la estructura del hogar son las variables con las que podemos acercarnos a la conexión entre curso vital e (in)movilidad. A través del régimen de tenencia de la vivienda, establecemos la conexión de los comportamientos con el estado en la carrera residencial (housing career). La condición socioeconómica individual muestra la relación con la posición de poder, así como con la situación de ventaja y desventaja en la estructura social. De la misma forma, el tipo de sección censal (más acomodadas o más deprimidas) informa de la posición de los individuos en la estructura socioespacial urbana. La caracterización social de las secciones se ha realizado en base a los resultados de un análisis de conglomerados con datos censales del año 2001 (variable clave: condición socioeconómica de los residentes en cada sección). Por último, se incluye una variable referida a la presencia de redes sociales en el barrio como factor de arraigo espacial. Dicha variable se ha construido en base a las preguntas referidas a la localización de amigos y familiares externos al hogar. En el caso de disponer de redes informales y/o familiares en el barrio, nuestra variable dicotómica adquiere el valor 1 (para una descripción de las variables, ver la tabla 1 ).

Antes de ejecutar los modelos, se ha testado la existencia de multicolinealidad (usando el VIF test - variance inflation factors for the independent variables-) y la falta de especificación (Ramsey regression specification-error test) entre las variables para asegurar la consistencia de los resultados y de las predicciones. A su vez, aunque los test de heterocedasticidad no indican problemas significativos, los modelos se han ejecutado aplicando robustez en el cálculo de errores estándar (VCE Robust) ${ }^{1}$.

1. Para una explicación más completa de los test aplicados y su pertinencia, consultar Cameron y Trivedi, 2013. 
Tabla 1. Descripción de las variables

\begin{tabular}{|c|c|c|c|c|}
\hline \multirow[b]{2}{*}{ Variables dependientes } & \multicolumn{2}{|c|}{ (In)movilidad residencial } & \multicolumn{2}{|c|}{ (In)movilidad espacial } \\
\hline & $\%$ & Frecuencia & $\%$ & Frecuencia \\
\hline \multicolumn{5}{|l|}{ Número de movimientos (últimos 10 años) } \\
\hline 0 & 55,99 & $(1.126)$ & 34,92 & (309) \\
\hline 1 & 34,66 & (697) & 52,09 & (461) \\
\hline 2 & 6,96 & $(140)$ & 9,72 & (86) \\
\hline 3 & 1,74 & (35) & 2,15 & (19) \\
\hline 4 & 0,30 & (6) & 0,45 & (4) \\
\hline 5 & 0,30 & (6) & 0,68 & (6) \\
\hline 6 & 0,05 & (1) & & \\
\hline \multicolumn{5}{|l|}{ Variables independientes } \\
\hline Continuas & Media & D. E. & Media & D. E. \\
\hline Edad & 47,75 & $(17,82)$ & 40,48 & $(14,44)$ \\
\hline Categóricas & $\%$ & Frecuencia & $\%$ & Frecuencia \\
\hline \multicolumn{5}{|l|}{ Posición socioeconómica } \\
\hline Empresarios o profesionales & 25,46 & (512) & 26,89 & (238) \\
\hline Trabajadores administrativos (ref.) & 13,03 & (262) & 13,79 & (122) \\
\hline Trabajadores de los servicios & 18,05 & (363) & 19,77 & (175) \\
\hline Trabajadores manuales & 28,54 & (574) & 28,36 & (251) \\
\hline Otros y no clasificables & 14,92 & $(300)$ & 11,19 & (99) \\
\hline \multicolumn{5}{|l|}{ Estructura de hogar } \\
\hline Unipersonal & 10,49 & (211) & 11,19 & (99) \\
\hline Parejas (ref.) & 22,82 & (459) & 20,79 & (184) \\
\hline Familias & 35,01 & (704) & 48,81 & (432) \\
\hline Otros hogares & 31,68 & $(637)$ & 19,21 & $(170)$ \\
\hline \multicolumn{5}{|l|}{ Tenencia de vivienda } \\
\hline Propietarios, totalmente pagada & 52,01 & $(1.046)$ & 20,56 & (182) \\
\hline Propietarios con pagos pendientes (ref.) & 32,97 & (663) & 56,16 & $(497)$ \\
\hline Alquiler & 12,33 & (248) & 20,79 & (184) \\
\hline Otras formas & 2,69 & (54) & 2,49 & (22) \\
\hline Redes sociales en el barrio (ref.: Sin redes) & 36,55 & $(735)$ & 30,51 & $(270)$ \\
\hline \multicolumn{5}{|l|}{ Tipo de sección censal } \\
\hline Secciones acomodadas & 9,40 & (189) & 7,57 & (67) \\
\hline Secciones de clases medias (ref.) & 17,26 & (347) & 14,58 & (129) \\
\hline Secciones de clases medias mixtas & 26,16 & (526) & 25,65 & (227) \\
\hline Secciones populares & 27,65 & (556) & 28,25 & (250) \\
\hline Secciones deprimidas & 8,20 & (165) & 8,36 & (74) \\
\hline Nuevas secciones & 11,34 & (228) & 15,59 & (138) \\
\hline Total $(N)$ & & (2.011) & & (885) \\
\hline
\end{tabular}

Fuente: Ayuntamiento de Granada. Encuesta sobre vivienda y población metropolitana, 2008. 


\subsection{Técnicas y procedimiento}

La técnica básica de este artículo es la regresión de conteo. Muy extendida en ciencias de la salud o en el campo de la economía, ha sido menos utilizada para estudios de movilidad. A falta de bases de datos longitudinales con las que desarrollar nuevas estrategias, es una técnica que permite establecer mejores predicciones que la regresión lineal múltiple cuando la variable dependiente representa el número de veces que se repite un determinado fenómeno en el tiempo (Agresti, 2001; Cameron y Trivedi, 2013). Sin embargo, los distintos tipos de regresión de conteo parten de asunciones conceptuales diferentes. Por ello, una cuidada elección del modelo que mejor se ajuste a la realidad de nuestros datos es un paso necesario. Los cuatro tipos de regresión de conteo que aquí examinamos son: el modelo de regresión Poisson, el modelo de regresión binomial negativa, el modelo de regresión inflado en ceros y el modelo de regresión Hurdle (para una revisión del procedimiento, véase Cameron y Trivedi, 2013).

Básicamente, hay dos pasos en la elección. En primer lugar, comparamos los modelos clásicos: la regresión Poisson (PRM) y la binomial negativa (NBREG). La regresión Poisson, más ampliamente utilizada, funciona mejor cuando la distribución de la variable de conteo no presenta rasgos de sobredispersión (varianza condicional mayor que la media) y cuando tratamos con muestras pequeñas. Sin embargo, uno de los problemas a los que se enfrenta es que no tiene en cuenta la incidencia de varianza no observada en el modelo, lo cual puede provocar errores de predicción en las categorías modales de la variable dependiente. Esta es la mejora de la regresión binomial negativa con respecto a la regresión Poisson. La inclusión de un factor Alpha $(\alpha)$ no constante y que varía entre las variables incluidas en el modelo. Esta primera comprobación sirve para establecer qué tipo de acercamiento analítico se adecúa mejor a la hora de estudiar las trayectorias de (in)movilidad residencial y espacial con nuestra muestra y marcará el procedimiento a seguir en el diseño de los modelos de regresión de conteo posteriores.

En el segundo paso, incluimos en la comparación los modelos de regresión de ceros inflados (ZIP o ZINB) y los modelos Hurdle (HPLOGIT o HNBLOGIT). Ambos son ampliaciones de los modelos tradicionales y ambos intentan lidiar con una posible acumulación en el recuento 0 , en nuestro caso, no haberse movido en el periodo analizado. La regresión de conteo inflada en 0 parte de que, en la muestra, hay dos tipos de ceros, los verdaderos y un exceso de observaciones que responden a personas con nulas o escasas probabilidades de movilidad (recuentos mayores que 0 ). Aplica una ecuación para conocer la existencia y los factores implicados en el exceso de 0 y otra diferente para generar las predicciones de los verdaderos 0 conjuntamente con el resto de recuentos positivos. El modelo Hurdle parte de la idea de que la ocurrencia del fenómeno analizado se explica de forma distinta a la intensidad con la que se produce. Para ello, el modelo se compone de dos fracciones: un análisis de regresión logística binaria para calcular la probabilidad de tener 0 recuentos 
frente a tener 1 o más recuentos, y una regresión de conteo truncada en 0 para predecir los recuentos positivos (1 cambio a más). La comprobación del ajuste de este tipo de modelos genera un resultado interesante de cara a nuestros objetivos de investigación. Si el modelo Hurdle predice mejor la realidad observada, podemos argumentar que, en el estudio de la (in)movilidad residencial y espacial, hay dos cuestiones implicadas, cada una de las cuales está diferentemente afectada por las variables independientes: (1) moverse o quedarse, (2) moverse más o menos.

Para la selección final de la técnica analítica a utilizar, nos basamos en dos criterios:

a) La comparación de las medidas de ajuste de cada modelo².

b) La comparación entre las probabilidades predichas por los modelos y las probabilidades observadas para conocer con qué procedimiento cometemos más errores y en qué recuentos.

Una vez seleccionado el modelo que realiza predicciones más ajustadas, para analizar la relación de los factores explicativos con nuestras variables dependientes (segunda pregunta de investigación), presentamos los resultados de los modelos escogidos. Nos interesa saber qué características están significativamente conectadas con un comportamiento más o menos sedentario en términos residenciales y espaciales. Pero, también, cuáles son las diferencias existentes entre los factores en cada recuento. Para ello, calculamos las probabilidades específicas que tiene cada perfil (por ejemplo: propietarios) de acometer desde 0 hasta 3 cambios domiciliarios o espaciales. Debido a las diferencias de escala entre los recuentos (es bastante más frecuente moverse entre $0-2$ veces), hemos construido un índice específico (que se expresa en base 100) que elimine el efecto de la estructura de los recuentos y posibilite una mejor visualización de las diferencias entre las categorías y los factores (las tablas A-1 y A-2 -anexos- recogen las probabilidades predichas reales con las que se ha construido el índice). Siguiendo el ejemplo de los propietarios, este índice se ha calculado como sigue: probabilidad predicha que tienen los propietarios de recuento ${ }_{\mathrm{x}} /$ probabilidad predicha media de recuento ${ }_{\mathrm{x}}$. Este procedimiento se repite para cada categoría y para cada recuento.

\section{Resultados}

\subsection{Ajustando un modelo de análisis para el estudio de la (in)movilidad como historia de sucesos}

Para resolver nuestro primer objetivo de investigación - ¿cómo estudiar las historias de (in)movilidad con datos retrospectivos de conteo?-, en este apar-

2. Para conocer qué modelo realiza mejores predicciones, presentamos las medidas más ampliamente utilizadas en la comparación de modelos de regresión: LR Test, el criterio de información Akaike (AIC) y el criterio de información bayesiana (BIC). 
tado resumimos los resultados de la comparación entre los distintos modelos para cada una de nuestras variables dependientes. En la tabla 2, comparamos las medidas de ajuste de los cuatro modelos de regresión de conteo para la (in) movilidad residencial (domiciliaria). En cuanto al primer paso en dicha comparación, el modelo basado en una asunción Poisson resulta más adecuado que un modelo de regresión binomial negativa. Por dos motivos. Por un lado, la variable sobre cambios domiciliarios no presenta rasgos de sobredispersión (test de dispersión presentado en la tabla 2), más bien al contrario. En Granada, en un periodo de 10 años, el 56\% de los encuestados no realizó ningún movimiento, cerca del $35 \%$ realizó un cambio y solo algo menos del $10 \%$ realizó más de dos movimientos residenciales (tabla 1).

Por otro lado, la principal diferencia entre la regresión Poisson y la binomial negativa - la inclusión de un factor que aborde la varianza no observa$\mathrm{da}$ - no supone ninguna ventaja en términos analíticos (Alpha es tan cercano a 0 que no se puede determinar que no sea 0: hipótesis nula del test). En la misma dirección, el resto de medidas incluidas en la tabla 2 (criterio de información bayesiana - BIC - como el criterio de información de Akaike - AIC_-) varían escasamente entre ambos procedimientos, indicando que adoptar la asunción de la que parte la regresión binomial negativa no mejora las predicciones que realizamos sobre la (in)movilidad residencial, sino que las empeora levemente. Por ello, en la generación de los dos modelos restantes (regresión inflada en ceros y Hurdle), utilizamos asunciones Poisson.

El modelo de regresión inflado en ceros (tabla 2) tampoco supone mejoras significativas frente a la regresión de conteo Poisson. De hecho, el ajuste del modelo empeora (AIC, BIC y diferencia entre el modelo nulo-lleno). En nuestro caso, dichos resultados indican que, teniendo en cuenta las variables explicativas incluidas, no hay evidencias de que estén relacionadas con un exceso de ceros, por lo que adoptar un modelo que presupone la existencia de recuentos inflados en dicha categoría no resulta un abordaje adecuado. El

Tabla 2. Comparación de las medidas de ajuste para la variable (in)movilidad residencial

\begin{tabular}{lcc}
\hline & Reg. Poisson -PRM- & Reg. binomial negativa -NBREG- \\
\hline Log-likelyhood modelo completo & $-1616,989$ & $-1616,997$ \\
AIC & 3267,977 & 3267,995 \\
BIC & 3363,286 & 3363,304 \\
Poisson goodness-of-fit -poisgof- & No hay sobredispersión & \\
Likelihood ratio test (alpha) -LR Test & & Alpha no es sig. distinto de 0 \\
$N$ & 2.011 & 2.011 \\
\hline & Reg. zero inflated -ZIP- & Reg. Hurdle - LOGIT+ZTP- \\
\hline Log-likelyhood modelo completo & $-1607,812$ & $-1480,445$ \\
AIC & 3281,623 & 3028,889 \\
BIC & 3466,634 & 3219,507 \\
$N$ & 2.011 & 2.011 \\
\hline
\end{tabular}

Fuente: Ayuntamiento de Granada. Encuesta sobre vivienda y población metropolitana. 2008. 
Tabla 3. Comparación de las medidas de ajuste para la variable (in)movilidad espacial

\begin{tabular}{lcc}
\hline & Reg. Poisson -PRM- & Reg. binomial negativa -NBREG- \\
\hline Log-likelyhood modelo completo & $-970,048$ & $-974,906$ \\
AIC & 1978,097 & 1979,813 \\
BIC & 2069,023 & 2051,597 \\
Poisson goodness-of-fit -poisgof- & No hay sobredispersión & \\
Likelihood ratio test (alpha) -LR Test- & & Alpha no es sig. distinto de 0 \\
$N$ & 885 & 885 \\
\hline & Reg. zero inflated -ZIP- & Reg. Hurdle - LOGIT + ZTP- \\
\hline Log-likelyhood modelo completo & $-970,048$ & $-862,251$ \\
AIC & 1990,097 & 1800,503 \\
BIC & 2109,736 & 1982,356 \\
$N$ & 885 & 885 \\
\hline
\end{tabular}

Fuente: Ayuntamiento de Granada. Encuesta sobre vivienda y población metropolitana. 2008.

modelo Hurdle, sin embargo, muestra resultados más favorables. Este modelo es el que mejores medidas de ajuste presenta (AIC, BIC, modelo nulo-lleno). Es decir, disponiendo del total de cambios residenciales en un periodo de tiempo y teniendo en cuenta las dimensiones básicas - factores incluidos en el modelo-, es conveniente utilizar un procedimiento que adopte la asunción de partida del modelo en dos pasos Hurdle.

La comparación de los modelos de regresión de conteo cuando la variable dependiente es el número de cambios realizados fuera del barrio por los móviles ((in)movilidad espacial) arroja resultados muy similares a los descritos en párrafos anteriores. Los recuentos no están tan concentrados como lo estaban en la variable sobre cambios domiciliarios, pero siguen existiendo dos categorías modales y poca dispersión en la distribución de las observaciones. El 34,9\% de los móviles nunca han salido de su entorno inmediato en los últimos diez años. El $52,1 \%$ ha traspasado la barrera del barrio en sus trayectorias de movilidad una sola vez. El 13,4\% ha realizado dos o más cambios entre barrios (tabla 1 y test de sobredispersión en tabla 3). El resto de medidas de ajuste confirman los mismos resultados que advertimos respecto a la (in)movilidad domiciliaria: el mejor ajuste del modelo basado en asunciones Poisson ${ }^{3}$.

La regresión inflada en ceros es, de nuevo, la que peor se adapta a la realidad de nuestros datos sobre (in)movilidad espacial. Si hubiésemos incluido, en el análisis de dicha (in)movilidad entre barrios, a los individuos que no han cambiado de domicilio, un modelo inflado en ceros sí hubiese sido más adecuado, dado que todos aquellos que no se han movido de sus viviendas nunca tuvieron

3. Sin embargo, en el caso de la variable (in)movilidad espacial, en la misma tabla 3, comprobamos que el criterio de información bayesiana es contradictorio con el resto de indicadores y parece mostrar que el procedimiento NBREG sería una mejor opción. Pero los puntos de mejora son demasiado escasos y el criterio de información Akaike se ha demostrado más adecuado para comparar modelos con una misma muestra pero con diferentes procedimientos de análisis (Burnham y Anderson, 2004). 
la opción de cambiar de barrio. Pero, dado que no incluimos a la población que nunca tuvo la opción (0 cambios residenciales), este método se muestra poco adecuado (tabla 3). La asunción de partida del modelo Hurdle vuelve a resultar la más idónea para describir las relaciones entre la (in)movilidad espacial y nuestras variables independientes.

La figura 2, en la que se muestran las diferencias entre las probabilidades observadas y las predichas por los modelos PRM, ZIP y Hurdle, confirma los resultados mostrados en las tablas 2 y 3 . Tanto en la variable sobre (in)movilidad residencial (figura 2, arriba) como espacial (figura 2, abajo), los modelos

Figura 2. Diferencias entre probabilidades observadas y predichas por los modelos (arriba: movilidad residencial; abajo: movilidad espacial)
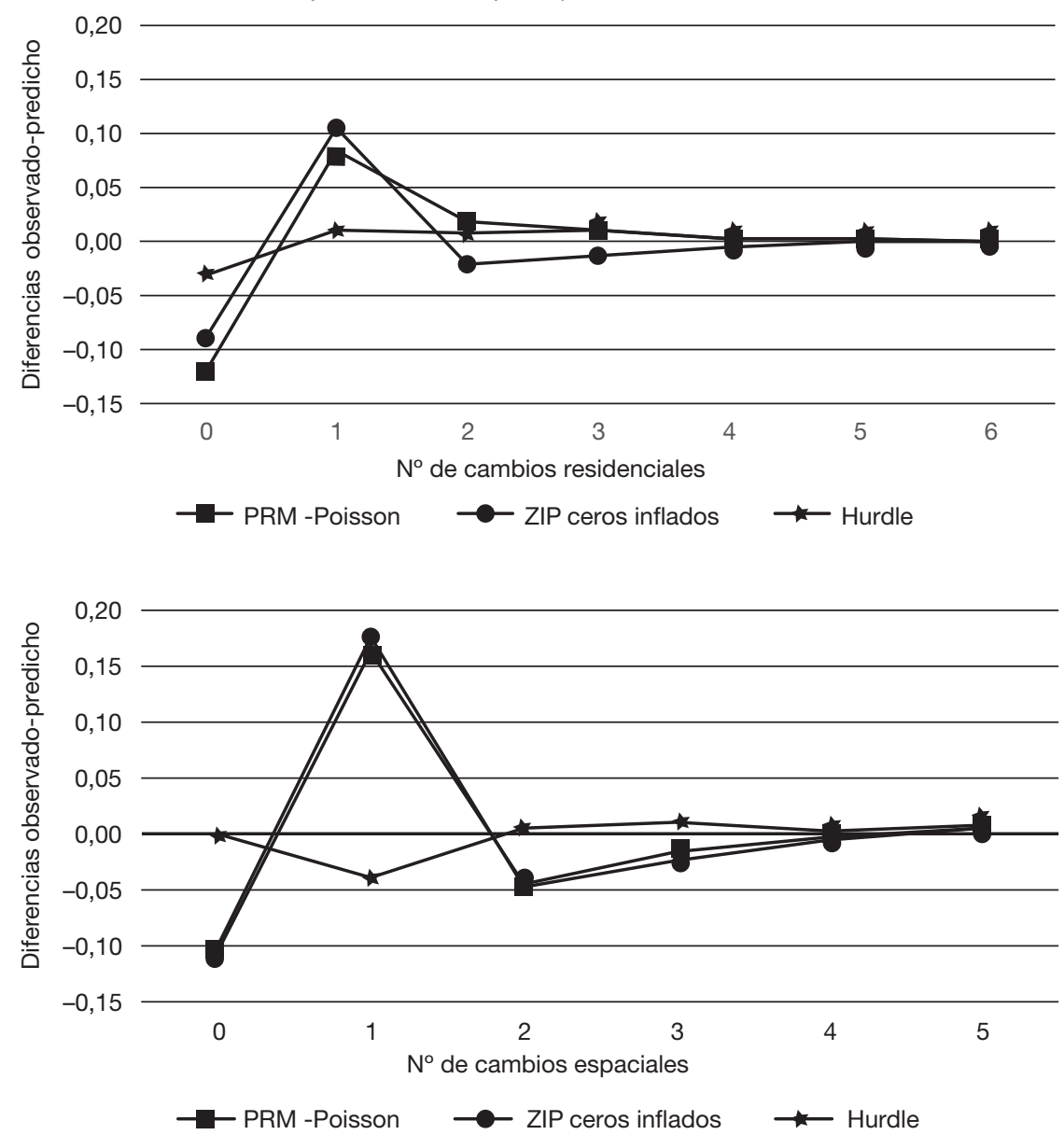

Fuente: Ayuntamiento de Granada. Encuesta sobre vivienda y población metropolitana. 2008. 
Poisson y de ceros inflados cometen más errores en la predicción de todos los recuentos, sobre todo en las dos categorías modales. Ambos procedimientos tienden a subestimar la inmovilidad (0 recuentos) y a subestimar la probabilidad de haber realizado 1 solo cambio en cerca de un 10\%. El modelo Hurdle realiza mejores predicciones. Aunque subestima los recuentos de la categoría modal de ambas variables dependientes, el error no supera el 5\% (0,05 puntos de diferencia entre observado y predicho).

Del análisis comparativo, concluimos que el modelo de regresión de conteo Hurdle es el más acertado para analizar las trayectorias de (in)movilidad residencial y espacial cuando la información disponible es el número de cambios en un periodo de tiempo. Como dijimos en secciones anteriores, la comparación entre modelos tiene fines analíticos en cuanto la elección de la mejor estrategia es un paso necesario para descubrir la verdadera relación existente entre las trayectorias de (in)movilidad y los factores explicativos en nuestro estudio. Pero pone de relieve que, contando con historiales de cambios residenciales y espaciales, la ocurrencia de inmovilidad (frente a la movilidad) no se explica de la misma forma que la intensidad con la que se producen las trayectorias a lo largo del periodo y del espacio analizados.

\subsection{Dimensiones básicas de (in)movilidad residencial y espacial}

\section{Resultados del modelo de regresión Hurdle}

La tabla 4 recoge los coeficientes, así como la significatividad con la que cada factor explicativo está asociado a la variable de recuento referida a la (in)movilidad residencial. Las dos primeras columnas tras los enunciados corresponden al modelo de regresión logística con el que predecimos la probabilidad de haber realizado cero cambios (valor 1 ), frente a haberse movido al menos una vez (valor 0). Las dos columnas siguientes muestran los resultados de la regresión de conteo Poisson para analizar la intensidad de las trayectorias de aquellos que hayan protagonizado uno o más movimientos (regresión Poisson truncada en ceros ZTP). Una primera revisión de las medidas de ajuste de ambas partes del modelo Hurdle (aportadas en las últimas filas de la tabla 4) indican que las variables independientes añadidas funcionan como buenas predictoras de la (in)movilidad residencial. Es decir, realmente son dimensiones básicas en el estudio de las historias de (in)movilidad residencial. Tanto la pseudo $\mathrm{R}^{2} \mathrm{de}$ Nagelkerke, por encima de 0,310 tanto en la regresión Logit como en la parte ZTP, como la diferencia entre el modelo completo respecto al nulo y el criterio de información Akaike, señalan que, tan solo con las dimensiones tenidas en cuenta, conseguimos una buena aproximación explicativa al fenómeno.

En el modelo Logit, los factores significativamente asociados a la probabilidad de quedarse (0 cambios domiciliarios) son rasgos bien documentados como características conectadas con las decisiones de (in)movilidad, pero también observamos asociaciones que pudieran deberse a características propias del contexto residencial español y, más concretamente, del contexto metropolitano de Granada. La edad está positivamente asociada a la inmovilidad. A medida que 
Tabla 4. Resultados de la regresión de conteo con el modelo Hurdle. Variable (in)movilidad residencial

\begin{tabular}{|c|c|c|c|c|}
\hline & \multicolumn{2}{|c|}{ Modelo Logit } & \multicolumn{2}{|c|}{ Modelo ZTP } \\
\hline & $B$ & S.E. & $B$ & S.E. \\
\hline Edad & $0,038^{\star \star \star}$ & $(0,004)$ & $-0,032^{\star \star \star}$ & $(0,005)$ \\
\hline \multicolumn{5}{|l|}{ Estructura de hogar (ref.: Parejas) } \\
\hline Unipersonal & $-0,113$ & $(0,221)$ & $0,361^{*}$ & $(0,194)$ \\
\hline Familias & $0,507^{\star \star \star}$ & $(0,168)$ & 0,028 & $(0,166)$ \\
\hline Otros hogares & $1,245^{\star \star \star}$ & $(0,179)$ & $-0,170$ & $(0,215)$ \\
\hline \multicolumn{5}{|c|}{ Tenencia de vivienda (ref.: Propietarios con pagos pendientes) } \\
\hline Propietarios sin cargas & $2,226^{\star \star \star}$ & $(0,135)$ & $-0,383$ & $(0,258)$ \\
\hline Alquiler & $-0,209$ & $(0,193)$ & $0,928^{\star \star \star}$ & $(0,144)$ \\
\hline Otras formas & $1,573^{\star \star \star}$ & $(0,339)$ & $-0,158$ & $(0,525)$ \\
\hline \multicolumn{5}{|l|}{ Posición socioeconómica (ref.: Trab. adm.) } \\
\hline Empresarios o profesionales & 0,238 & $(0,190)$ & $0,403^{\star \star}$ & $(0,201)$ \\
\hline Trabajadores de los servicios & 0,150 & $(0,207)$ & $0,424^{*}$ & $(0,217)$ \\
\hline Trabajadores manuales & 0,182 & $(0,195)$ & 0,042 & $(0,21)$ \\
\hline Otros y no clasificables & $0,404^{*}$ & $(0,214)$ & 0,282 & $(0,284)$ \\
\hline Redes sociales en el barrio (ref.: Sin redes en barrio) & $0,390^{\star \star \star}$ & $(0,126)$ & $-0,219$ & $(0,164)$ \\
\hline \multicolumn{5}{|l|}{ Tipo de sección censal (ref.: Secciones clases medias) } \\
\hline Secciones acomodadas & $-0,044$ & $(0,244)$ & $-0,604^{\star \star}$ & $(0,220)$ \\
\hline Secciones de clases medias mixtas & $-0,246$ & $(0,234)$ & $-0,258$ & $(0,296)$ \\
\hline Secciones populares & $-0,595^{\star \star \star}$ & $(0,181)$ & $-0,455^{\star \star}$ & $(0,172)$ \\
\hline Secciones deprimidas & $-0,426^{\star}$ & $(0,191)$ & $-0,457^{\star \star}$ & $(0,184)$ \\
\hline Nuevas secciones & $-0,730^{\star \star \star}$ & $(0,254)$ & $-0,208$ & $(0,255)$ \\
\hline Constante & $-3,177^{\star \star \star}$ & $(0,34)$ & 0,283 & $(0,347)$ \\
\hline$N$ & 2.011 & & 885 & \\
\hline Log-ver. Solo constante & $-1379,443$ & & $-780,174$ & \\
\hline Log-ver. Modelo completo & $-942,998$ & & $-531,554$ & \\
\hline Pseudo $\mathrm{R}^{2}$ & 0,316 & & 0,319 & \\
\hline AlC & 1921,996 & & 1099,108 & \\
\hline
\end{tabular}

Fuente: Ayuntamiento de Granada. Encuesta sobre vivienda y población metropolitana, 2008.

las personas se adentran en etapas maduras del curso vital, la probabilidad de no haber realizado ningún cambio se incrementa. Con respecto a los hogares habitados por parejas (referencia), las familias, así como otros hogares que no conforman núcleos, tienen también una mayor probabilidad de no haberse movido. Las personas solas, sin embargo, no son más propensas a la movilidad que las parejas, dato que contradice resultados obtenidos en contextos internacionales, pero que responde a la relevancia del matrimonio o del emparejamiento como desencadenante de gran parte de los cambios domiciliarios de emancipación (Holdsworth y Irazoqui, 2002). Tal como esperábamos por los estudios desarrollados en otros países, así como por la cultura de tenencia característica de España, ser propietario sin pagos pendientes, así como residir 
en viviendas cedidas $\mathrm{u}$ otras formas de tenencia minoritarias, son las características más intensamente conectadas con el sedentarismo.

La condición socioeconómica, por el contrario, no apunta grandes diferencias. Existe una asociación positiva entre la inmovilidad y la categoría de otros y no clasificables, que engloba perfiles como pensionistas por invalidez o sin trabajo remunerado, indicando que el sedentarismo está conectado con las situaciones de dependencia económica o vulnerabilidad laboral. Pero, aparte de dicha conexión, cuando lo que se analiza es la ocurrencia de (in)movilidad residencial en un periodo de tiempo, las clases sociales más vulnerables no se muestran más sedentarias que otras clases mejor posicionadas en la estructura socioeconómica. La presencia de redes sociales y familiares en el entorno inmediato del barrio, tal como apuntaban los estudios desarrollados por Dawkins (2006) y Clark et al. (2015), funciona como potente factor de arraigo. Vivir rodeado de familiares y amigos aumenta la probabilidad de que los individuos permanezcan en sus domicilios. El tipo de sección censal, que sirve como indicador del carácter social del entorno inmediato del barrio, marca también diferencias. Existe una asociación negativa y estadísticamente significativa entre residir en barrios nuevos, populares y deprimidos y movilidad. El sedentarismo no es más probable en zonas vulnerables, justo al contrario, es más improbable.

En cuanto al análisis de la intensidad de las trayectorias (ZTP, dos últimas columnas de la tabla 4), la edad vuelve a indicar que los jóvenes tienden a desarrollar historias más móviles. Las personas que viven solas no tienen una mayor probabilidad que las parejas de moverse, pero sí tienen una conexión significativamente positiva con la hipermovilidad. Es más probable que realicen más movimientos que el resto de hogares. La residencia en alquiler es el factor más fuertemente ligado a la alta movilidad. La condición socioeconómica, al contrario de lo que sucede en el modelo Logit, sí marca diferencias significativas. Los profesionales y trabajadores de los servicios son perfiles con mayor probabilidad de realizar historias más móviles dentro del área metropolitana. Por último, la influencia de los factores socioespaciales en la intensidad de la movilidad también varía con respecto al modelo Logit. La presencia de redes sociales no es estadísticamente relevante, pero sí lo es el tipo de barrio. La residencia en barrios acomodados, populares y deprimidos tiene una relación negativa con la hipermovilidad. Es decir, sus habitantes tienden a moverse menos veces que los residentes en otro tipo de barrios. Estos resultados advierten de las diferencias que afloran cuando analizamos historias de movilidad completas. Cuando se dispone de datos referidos a la intensidad de la movilidad, la posición en la estructura social y urbana es un factor relevante para explicar comportamientos más o menos sedentarios.

En la tabla 5, presentamos el modelo de regresión Hurdle aplicado a nuestra segunda variable dependiente: la (in)movilidad espacial. Las medidas de ajuste apuntan que el modelo ZTP predice más acertadamente que el modelo Logit. Es decir, las variables independientes incluidas explican bien la intensidad de la movilidad entre barrios, pero no son buenas predictoras del sedentarismo espacial. Por ello, un primer análisis del ajuste indica que, para conocer 
Tabla 5. Resultados de la regresión de conteo con el modelo Hurdle. Variable (in)movilidad espacial

\begin{tabular}{|c|c|c|c|c|}
\hline & \multicolumn{2}{|c|}{ Modelo Logit } & \multicolumn{2}{|c|}{ Modelo ZTP } \\
\hline & $B$ & S.E. & $B$ & S.E. \\
\hline Edad & $-0,006$ & $(0,005)$ & $-0,033^{\star \star \star}$ & $(0,008)$ \\
\hline \multicolumn{5}{|l|}{ Estructura de hogar (ref.: Parejas) } \\
\hline Unipersonal & $-0,294$ & $(0,299)$ & $0,482^{*}$ & $(0,276)$ \\
\hline Familias & 0,131 & $(0,196)$ & $-0,036$ & $(0,24)$ \\
\hline Otros hogares & 0,360 & $(0,228)$ & $-0,147$ & $(0,302)$ \\
\hline \multicolumn{5}{|l|}{ Tenencia de vivienda (ref.: Propietarios con pagos pendientes) } \\
\hline Propietarios sin cargas & $0,382^{\star \star}$ & $(0,198)$ & $-0,882^{\star \star}$ & $(0,373)$ \\
\hline Alquiler & $0,407^{\star \star}$ & $(0,201)$ & $0,790^{\star \star \star}$ & $(0,179)$ \\
\hline Otras formas & 0,675 & $(0,434)$ & $-0,320$ & $(0,623)$ \\
\hline \multicolumn{5}{|l|}{ Posición socioeconómica (ref.: Trab. administrativos) } \\
\hline Empresarios o profesionales & $-0,422^{\star}$ & $(0,239)$ & $0,565^{\star \star}$ & $(0,283)$ \\
\hline Trabajadores de los servicios & $-0,325$ & $(0,249)$ & 0,356 & $(0,33)$ \\
\hline Trabajadores manuales & $0,403^{*}$ & $(0,226)$ & $-0,387^{\star \star}$ & $(0,263)$ \\
\hline Otros y no clasificables & $-0,348$ & $(0,292)$ & 0,439 & $(0,383)$ \\
\hline Redes sociales en el barrio (ref.: Sin redes en el barrio) & $0,974^{\star \star \star}$ & $(0,16)$ & $-0,048$ & $(0,232)$ \\
\hline \multicolumn{5}{|l|}{ Tipo de sección censal (ref.: Secciones clases medias) } \\
\hline Secciones acomodadas & $-0,261$ & $(0,286)$ & $-0,775^{\star \star}$ & $(0,259)$ \\
\hline Secciones de clases medias mixtas & $-0,174$ & $(0,361)$ & $-0,261$ & $(0,368)$ \\
\hline Secciones populares & 0,045 & $(0,261)$ & $-0,731^{\star \star \star}$ & $(0,211)$ \\
\hline Secciones deprimidas & 0,150 & $(0,26)$ & $-0,621^{*}$ & $(0,276)$ \\
\hline Nuevas secciones & $-0,292$ & $(0,346)$ & $-0,192$ & $(0,322)$ \\
\hline Constante & $-0,744^{\star}$ & $(0,397)$ & 0,351 & $(0,501)$ \\
\hline N & 885 & & 576 & \\
\hline Log-ver. Solo constante & $-572,525$ & & $-502,537$ & \\
\hline Log-ver. Modelo completo & $-531,277$ & & $-335,305$ & \\
\hline Pseudo $\mathrm{R}^{2}$ & 0,072 & & 0,333 & \\
\hline AIC & 1098,554 & & 706,609 & \\
\hline${ }^{*} p<0,10$, & & & & \\
\hline
\end{tabular}

Fuente: Ayuntamiento de Granada. Encuesta sobre vivienda y población metropolitana, 2008.

diferencias entre quienes no se mueven nunca de su contexto inmediato frente a los que lo hacen alguna vez, necesitaríamos incluir nuevas u otras variables explicativas. De hecho, observando los coeficientes y los errores estándar del modelo Logit (dos primeras columnas), comprobamos que tan solo tres variables tienen una relación estadísticamente significativa con la probabilidad de permanecer en el barrio siempre (inmovilidad espacial).

La edad, la estructura del hogar y el tipo de sección, que sí eran relevantes para explicar el sedentarismo residencial (no moverse del domicilio), no marcan diferencias en cuanto al sedentarismo espacial (moverse pero permanecer en el barrio). Sí lo hace la condición socioeconómica. Los profesionales y empre- 
sarios tienen una mayor probabilidad de realizar algún movimiento fuera del barrio. Los trabajadores manuales, por el contrario, tienen una mayor probabilidad de permanecer en el mismo barrio, dato que puede estar indicando las constricciones de salida que sus habitantes encuentran (van Ham y Clark, 2009). En cuanto al régimen de tenencia de la vivienda, llama la atención que tanto propietarios como personas en alquiler tienen una mayor tendencia a permanecer en el barrio frente a los propietarios con pagos pendientes. Dado que en el análisis de la (in)movilidad espacial solo hemos tenido en cuenta a las personas que efectuaron algún movimiento durante el periodo, este dato puede estar señalando que, tanto para asentamientos definitivos como temporales, el conocimiento y la experiencia del barrio puede ser un aspecto fundamental en la elección. De hecho, entre todos los factores significativamente asociados al sedentarismo espacial, tener redes sociales en el contexto inmediato es la variable más fuertemente ligada a la probabilidad de permanecer en el barrio.

Los resultados del modelo truncado en ceros (ZTP) muestran conexiones distintas a las que encontramos en los modelos Logit. La edad está inversamente relacionada con la probabilidad de realizar más cambios espaciales. Es decir, a menor edad, mayor probabilidad de efectuar más movimientos entre barrios. Las personas solas también tienen una mayor propensión a cambiar de barrio cuando cambian de residencia. El resto de hogares no tiene una relación significativamente distinta a la que tiene la categoría de referencia (parejas) con la intensidad de la movilidad espacial. Los propietarios tienden a realizar menos movimientos espaciales. Las personas en alquiler, al contrario de lo que ocurría en el modelo Logit, tienen una mayor probabilidad de realizar más cambios entre barrios.

La condición socioeconómica marca diferencias significativas y en la misma dirección que lo señalado en el modelo Logit. Las clases más vulnerables tienen una menor probabilidad de acometer cambios que conlleven cruzar la frontera de sus barrios, mientras que las clases profesionales acometen este tipo de cambios espaciales en mayor medida que el resto de clases sociales. Por último, el tipo de sección, no relevante en el modelo Logit (móviles siempre en el barrio/ al menos alguna vez fuera), sí lo es en el análisis de la intensidad con la que las personas cambian de espacio de vida específico. Residir en barrios acomodados, populares y deprimidos está relacionado con una menor propensión a efectuar movimientos fuera del barrio. Este dato, que más adelante discutiremos, señala la mayor propensión hacia un comportamiento sedentario en términos espaciales cuando se reside en zonas en ambos polos de la estructura socioespacial urbana.

\section{Principales diferencias en las probabilidades predichas para cada recuento ${ }^{4}$}

En la figura 3, presentamos gráficamente las diferencias en cuanto a las probabilidades predichas de las categorías de las variables explicativas para cada

4. Dado que la variable sobre redes sociales en el barrio es dicotómica, la visualización de las probabilidades no aporta ninguna información adicional a la señalada en los modelos. Por ello, no se representa gráficamente. 
recuento (expresadas en forma de índice — ver metodología-). Hemos calculado las probabilidades hasta un máximo de tres recuentos, dado que son pocos los individuos que realizan más cambios. En la misma figura, mostramos los resultados para la (in)movilidad residencial (izquierda) y espacial (derecha). De esta forma, además de comprobar las diferencias entre recuentos, también podemos hacer una reflexión sobre los distintos patrones encontrados entre los dos tipos de (in)movilidades estudiadas en este artículo. De hecho, revisando la misma figura 3 (y la tabla A.1 para comprobar los valores de las probabilidades predichas), hay dos primeras apreciaciones que afectan a ambas variables dependientes:

- Dado que la mayor parte de los individuos se concentran en los recuentos 0 y 1 cambio, su peso a la hora de determinar la significatividad de los modelos es desproporcionado. Por ejemplo, recordando los resultados de la regresión Hurdle para la (in)movilidad espacial (tabla 5), la edad así como otras variables explicativas no estaban significativamente asociadas a una mayor o menor propensión de permanecer en el barrio frente a moverse alguna vez fuera del mismo (modelo Logit), pero, observando la figura 3 , advertimos que, en realidad, donde no existen diferencias significativas es entre los que permanecen siempre en el barrio y los que han cruzado la frontera una sola vez. La edad, la estructura de hogar o el tipo de sección son factores que sí marcan diferencias claras a partir del segundo recuento. Por ello, una vez que descartamos a los sedentarios espaciales (siempre en el barrio), en el modelo truncado en ceros (ZTP), estos factores vuelven a recuperar su significatividad a la hora de explicar la intensidad de la movilidad.

- De la comparación entre (in)movilidad residencial y espacial, también traslucimos que la alta movilidad residencial parece estar asociada a una alta movilidad espacial. Es decir, los individuos que cambian más veces de domicilio tienden a hacerlo trazando trayectorias urbanas más amplias, cambiando más veces de barrio.

Continuando con el análisis más específico, en la misma figura 3 comprobamos que la movilidad residencial está asociada a etapas jóvenes, así como el sedentarismo lo está con etapas maduras. La movilidad es más probable durante la juventud y la primera etapa adulta, dada la concentración de acontecimientos desencadenantes que se producen en torno a estas edades, pero, sobre todo, destaca el protagonismo de los grupos comprendidos entre los 18 y los 39 años en la hipermovilidad. A pesar de encontrarse en etapas tempranas de la carrera residencial, cambian de domicilio más veces que los grupos mayores de 40 años. Fijándonos en las diferencias etarias de los móviles respecto a su comportamiento espacial (derecha, figura 3), las tendencias son similares pero menos marcadas. Los adultos, aunque son más sedentarios que los jóvenes en términos residenciales, no lo son en términos espaciales. Todos los grupos tienen una probabilidad similar de permanecer siempre en el barrio, señalando que, en la 
probabilidad de cambiar de barrio, entran aspectos explicativos más allá del curso vital, y más relacionados con las constricciones estructurales que afectan a las decisiones. La edad marca diferencias en el análisis de la intensidad con la que se cambia de barrio, pero solo entre los que se mueven de su contexto inmediato dos o más veces.

Los patrones referentes a la estructura de hogar son más claros. Tanto en términos residenciales como espaciales, las familias, así como otros hogares (no nucleares/no familiares), son los grupos asociados a un comportamiento más sedentario. Tienen una mayor probabilidad de no moverse, pero también de moverse menos que los hogares unipersonales (y que las parejas, categoría de referencia). En cuanto a las diferencias según régimen de tenencia, los propietarios sin cargas constituyen el perfil más sedentario. Las personas en régimen de alquiler son, por el contrario, los protagonistas absolutos de la movilidad. Las diferencias entre rentistas y propietarios se acentúan enormemente en recuentos altos. El alquiler, como modo de tenencia, está intrínsecamente ligado a la movilidad, pero sobre todo a la hipermovilidad.

El análisis de las diferencias según la condición socioeconómica revela lo que apuntaban los modelos de regresión y que queda oculto cuando solo se analiza la ocurrencia de movilidad domiciliaria versus la no ocurrencia de esta. Los grupos tienen una probabilidad similar de no moverse o de hacerlo una vez, pero las diferencias afloran cuando analizamos recuentos más altos. Los profesionales y trabajadores de los servicios tienden a moverse más. Sin embargo, los primeros suelen cruzar la frontera del barrio al mudarse en mayor medida que los segundos. Los trabajadores manuales son el grupo más sedentario. Tienen una menor probabilidad que el resto de moverse más de una vez. Pero además, cuando lo hacen, la probabilidad de permanecer siempre en el mismo barrio o traspasar sus fronteras una o más veces es bastante más escasa que para el resto de perfiles socioeconómicos.

Por último, las diferencias en las probabilidades predichas para cada tipo de sección censal indican tendencias singulares. Residir en zonas nuevas y medias mixtas está relacionado con una mayor movilidad residencial, pero también espacial. Las personas que viven en estos contextos cambian más veces de residencia y de barrio. En términos domiciliarios, los residentes en zonas acomodadas son los más sedentarios. Tienen una mayor probabilidad de no moverse ( 0 cambios) o de hacerlo en menor medida que habitantes de otras zonas. En términos espaciales, también desarrollan historias más sedentarias, pero, en este caso, el grupo con mayor tendencia a permanecer en el barrio es el compuesto por los habitantes de zonas deprimidas y populares. Aunque residir en estos barrios más vulnerables no determina una mayor predisposición al sedentarismo residencial absoluto (de hecho, es más probable que realicen 1 cambio domiciliario), sí determina historias residenciales menos intensas y, sobre todo, más localizadas en el contexto inmediato. 
Figura 3. Diferencias en las probabilidades predichas para cada recuento. Índice en base a la probabilidad predicha media de cada recuento
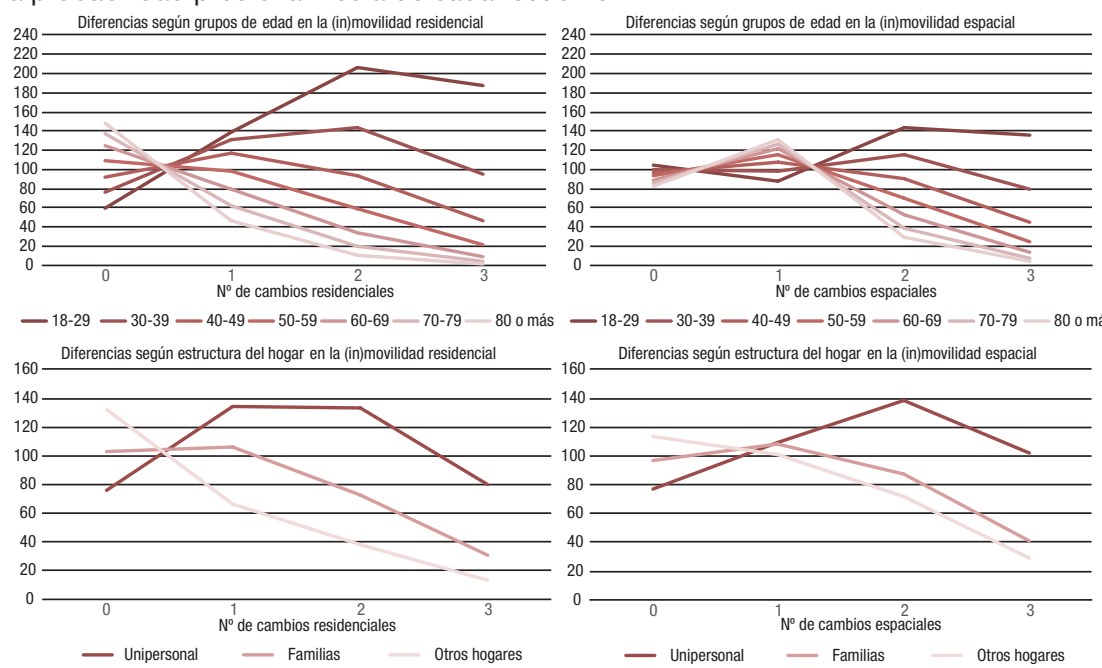

Diferencias según rég. tenencia en la (in)movilidad residencia

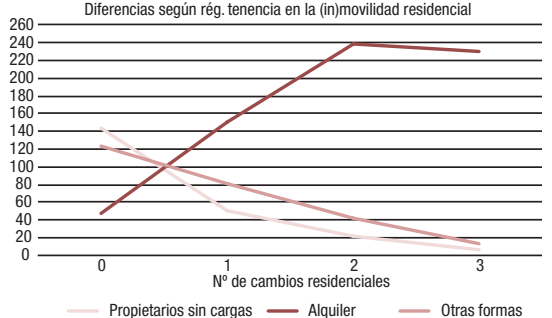

Diferencias según rég. tenencia en la (in)movilidad espacial

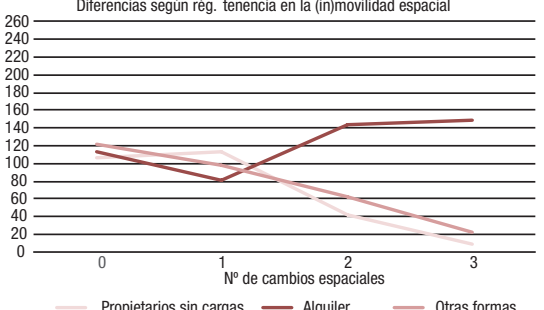

Diferencias segúncondición socioeconómica en la (in)movilidad residencial

Diferencias segúncondición socioeconómica en la (in)movilidad espacial
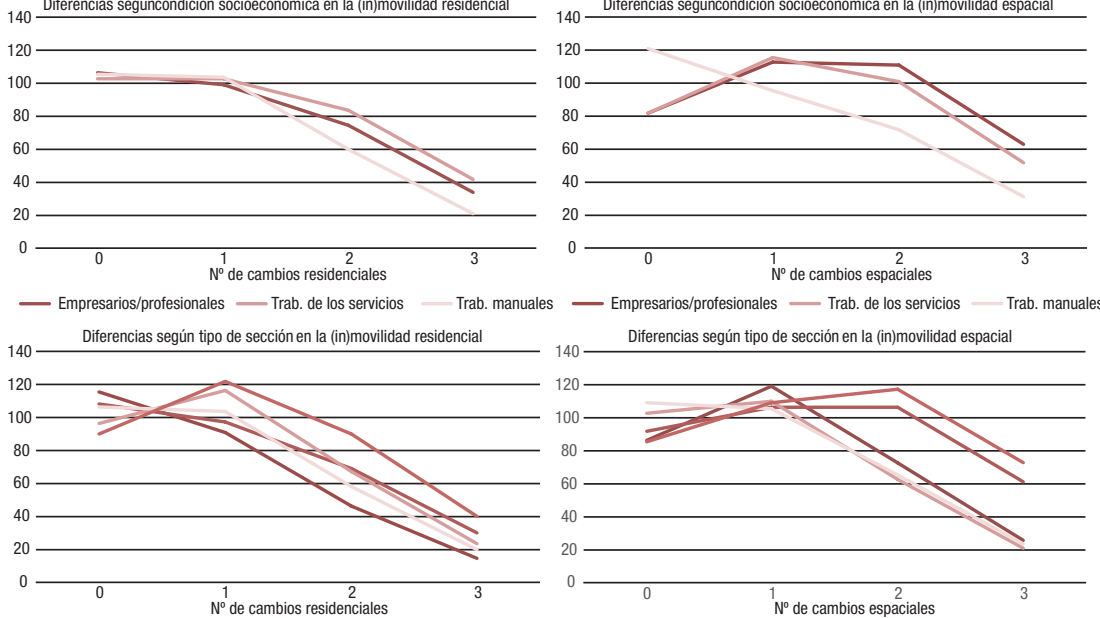

- Acomodadas — Medias mixtas — Populares - Deprimidas — Nuevas sec. — Acomodadas — Medias mixtas — Populares - Deprimidas — Nuevas sec.

Fuente: Ayuntamiento de Granada. Encuesta sobre vivienda y población metropolitana, 2008. 


\section{Discusión y conclusiones}

A pesar del bagaje acumulado en otros países, en España, las fuentes de microdatos disponibles en materia de movilidad han resultado insuficientes para afrontar análisis de las historias individuales residenciales y espaciales dentro de contextos urbanos 5 . Con base a una encuesta con información acerca de las trayectorias de (in)movilidad, este trabajo permite realizar una aproximación al estudio de dichas historias en un contexto metropolitano español. En todo caso, aunque los datos disponibles para el área metropolitana de Granada nos han permitido llevar a cabo un primer abordaje al estudio de las historias de (in)movilidad en contextos urbanos, para avanzar nuevos análisis, necesitaríamos disponer de fuentes con información más plural en cuanto a cuestiones que se recogen y más ricas en cuanto a su sistematización y periodicidad. Ya sea en forma de encuesta periódica, como el British Household Panel Survey (BHPS) usado para estudios desarrollados en el Reino Unido (Coulter y van Ham, 2013), o en base a registros administrativos longitudinales utilizados en países como Suecia (Fischer y Malmberg, 2001) o los Países Bajos (van Ham et al., 2014), la disposición de datos longitudinales (o paneles específicos sobre comportamiento residencial) posibilitaría corroborar algunos de los resultados obtenidos en este trabajo y es una apuesta indispensable si queremos profundizar en la explicación de la (in)movilidad en contextos urbanos y realizar comparaciones a lo largo del territorio español, así como a nivel internacional.

Ante la primera pregunta de nuestra investigación (¿Cómo estudiar historias de (in)movilidad residencial y espacial con los datos disponibles?), este artículo ofrece una estrategia analítica para abordar dicha cuestión con datos retrospectivos. Cuando se dispone de historias de movilidad completas durante un periodo, pero no de las decisiones ni de las características de los cambios y/o de las permanencias año a año (propio de fuentes longitudinales descritas en el párrafo anterior), los modelos de regresión de conteo Hurdle suponen una buena técnica con la que abordar el análisis. Si adoptásemos un análisis de regresión lineal o de conteo clásico, estaríamos cometiendo errores al asumir que el paso entre cada recuento — desde 0 cambios al máximo registrado— se produce siguiendo la misma lógica. En realidad, hay dos lógicas implicadas. Cuando se estudian las historias residenciales en términos domiciliarios y espaciales, hay un proceso de relación dominando la ocurrencia de inmovilidad frente a la ocurrencia de movilidad. Es decir, hay un proceso que determina el sedentarismo y otro proceso que domina el grado de intensidad con la que las historias de movilidad se producen. Aunque este tipo de modelos se ha corroborado como el idóneo en estudios sobre el comportamiento individual ante los sistemas de atención en salud (Salinas-Rodríguez et al., 2009), este artículo es el primero en comprobar que también se adaptan mejor que otros

5. La información recogida en censos - fuente estadística principal con microdatos disponibles - señala la existencia o la inexistencia de movimientos durante un periodo de tiempo - 1 año / 10 años-, pero: a) no recoge la totalidad de movimientos realizados en dicho periodo y $b$ ) no detalla el ámbito intramunicipal de los cambios. 
modelos para el estudio de los historiales de (in)movilidad urbana. Esperamos que, de esta investigación, surjan nuevos análisis en otras ciudades españolas para poder realizar los necesarios ejercicios comparativos.

En cuanto a nuestro segundo objetivo, conocer la relación entre (in)movilidad y los factores explicativos, existen diferentes aspectos a resaltar. El sedentarismo domiciliario está fuertemente conectado al transcurso vital y al estado en la carrera residencial, pero también tiene una alta asociación con los lazos establecidos en el contexto inmediato. Estos resultados, en la línea de otros estudios desarrollados en Granada (Clark et al., 2015), así como en otras ciudades europeas (Hjälm, 2014), confirman que el arraigo en términos de vida (etapas adultas y ancianas; familias consolidadas), de vivienda (propiedad) y de espacio (presencia de redes sociales en el barrio) está asociado a una mayor propensión hacia la inmovilidad. Tal como Fischer y Malmberg (2001) o Dawkins (2006) demostraron en otros contextos, los hogares con mayor grado de arraigo y con unos lazos espaciales más diversos, se mueven menos que otros hogares y se quedan por más tiempo en el mismo domicilio.

La posición en la estructura social no marca grandes diferencias, y los resultados según el tipo de sección advierten que la inmovilidad domiciliaria no es más esperable donde mayores constricciones se producen (entornos populares o deprimidos), sino en barrios con condiciones más favorables. Sin embargo, el efecto de dicha posición socioespacial adquiere otro signo a la hora de explicar qué determinados actores desarrollan trayectorias más sedentarias (menos intensas). A excepción de la presencia de redes sociales, cuya influencia se diluye cuando se analiza la intensidad de la movilidad, el arraigo en sus dimensiones de vida y vivienda siguen siendo una pieza fundamental. Pero la vulnerabilidad social emerge también como factor explicativo. Las clases más vulnerables y los residentes en barrios más desfavorecidos tienen una mayor probabilidad de desarrollar trayectorias sedentarias, compuestas, en muchos casos, por un solo movimiento. Los segundos y sucesivos cambios, en cuanto responden a motivos más relacionados con la mejora de las condiciones de vivienda, o con aspiraciones de movilidad social, afloran como más difícilmente abordables cuanto más baja es la posición socioespacial de partida. Aunque otros estudios referidos a la realidad española no han encontrado grandes diferencias sociales respecto a la movilidad (Susino y Palomares-Linares, 2013), en este artículo ponemos de relieve la existencia de dichas diferencias. Menos marcadas si solo se estudia la ocurrencia o no de movilidad, pero relevantes para entender las trayectorias en términos de intensidad de movimientos.

En cuanto al sedentarismo espacial, caben varias discusiones. Por un lado, el análisis del ajuste del modelo muestra que las dimensiones tenidas en cuenta son insuficientes para explicar la inmovilidad espacial. En futuros análisis y siempre que los datos lo posibiliten, debiéramos incluir otras características de relación con el barrio (variables más específicas sobre la localización de redes familiares o la actividad diaria desarrollada en el contexto del barrio). Pero también otras características de relación con el espacio urbano más amplio; por ejemplo: la situación geográfica del barrio dentro de la ciudad o las carac- 
terísticas edificatorias del contexto donde se reside. Como advertimos en el primer párrafo de esta sección, este tipo de acercamientos más profundos solo serán posibles en la medida en que se apueste por generar nuevas fuentes de datos con información geográfica suficiente para abordar análisis en contextos urbanos.

Por otro lado, con las dimensiones tenidas en cuenta en este trabajo, comprobamos la existencia de una intensa conexión entre inmovilidad espacial, estructura social y estructura urbana. Ciertamente, el sedentarismo espacial es más probable cuando se vive en ambos polos de la estructura socioespacial (zonas acomodadas y zonas deprimidas), apuntando de nuevo que la inmovilidad no siempre es fruto de la imposición, sino también de la capacidad de elección. Pero, en concordancia con estudios internacionales (Sabagh et al., 1969; Crowder y South, 2005; Clark et al., 2006), son las clases vulnerables y los residentes en zonas desfavorecidas los que muestran mayores dificultades a la hora de traspasar la frontera de sus barrios. Son más sedentarios en términos absolutos (mayor probabilidad de permanecer en el barrio todo el periodo analizado) y en términos relativos (menor probabilidad de realizar uno o más movimientos fuera del barrio).

En el polo opuesto, los patrones de alta movilidad residencial son adoptados por perfiles jóvenes, con pocas cargas familiares y, sobre todo, en situación de alquiler. A su vez, existe una sinergia entre intensidad de cambios domiciliarios y espaciales. Historias más móviles en términos residenciales conllevan frecuentes cambios dentro del espacio urbano. Las relaciones encontradas, tal como señalamos en apartados anteriores, sugieren que la hipermovilidad es un patrón que responde a nuevas formas de entender la movilidad residencial como estrategia vital (Aramburu, 2015) y que emerge y se consolida en paralelo a los cambios sociales, laborales y culturales de las últimas décadas.

\section{Referencias bibliográficas}

Agresti, Alan (2001). Categorical Data Analysis. 2. a ed. Nueva York: Wiley.

Aramburu, Mikel (2015). "Rental as a Taste of Freedom: The Decline of Home Ownership amongst Working class Youth in Spain during Times of Crisis». International Journal of Urban and Regional Research [en línea], 39 (6), 1172-1190. $<\mathrm{http}: / /$ dx.doi.org/10.1111/1468-2427.12218>.

BAYONA I CARRASCO, Jordi y LÓPEZ GaY, Antonio (2011). "Concentración, segregación y movilidad residencial de los extranjeros en Barcelona». Documents d’Anàlisi Geogràfica [en línea], 57 (3), 381-412. $<$ http://dx.doi.org/10.5565/rev/dag.234>.

BurnhaM, Kennet P. y ANDERSON, David R. (2004). «Multimodel inference: Understanding AIC and BIC in Model Selection». Sociological Methods \& Research [en línea], 33, 261-304. $<$ http://dx.doi.org/10.1177/0049124104268644>.

Cameron, Collin A. y Trivedi, Pravin K. (2013). Regression Analysis of Count Data [en línea]. Cambridge: Cambridge University Press. $<$ https://doi.org/10.1017/CBO9781139013567>. 
Campbell, Karen E.; Marsden, Peter V. y Hurlbert, Jeanne S. (1986). «Social resources and socioeconomic status». Social Networks [en línea], 8, 97-117. <http://dx.doi.org/10.1016/S0378-8733(86)80017-X>.

Chevan, Albert (1971). «Family growth, household density, and moving». Demography [en línea], 8, 451-458. <http://dx.doi.org/10.2307/2060682>.

Clark, William A.V. (1982). «Recent research on migration and mobility: A review and interpretation». Progress in Planning [en línea], 18, 1-56. <http://dx.doi.org/10.1016/0305-9006(82)90002-2>.

- (2013). "Life course events and residential change: Unpacking age effects on the probability of moving». Journal of Population Research [en línea], 30 (4), 319-334. <http://dx.doi.org/10.1007/s12546-013-9116-y>.

Clark, William A.V.; Deurloo, Marinus C.; Dieleman, Frans M. (2003). «Housing careers in the United States, 1968-93: Modelling the sequencing of housing states». Urban Studies [en línea], 40 (1), 143-160. <http://dx.doi.org/10.1080/00420980220080211>.

- (2006). «Residential mobility and neighbourhood outcomes». Housing Studies [en línea], 21 (3), 323-342. <http://dx.doi.org/10.1080/02673030600585946>.

Clark, William A.V. y Dieleman, Frans M. (1996). Households and housing: Choice and Outcomes in the Housing Market. New Brunswick: CUPR Press.

Clark, William. A.V.; Duque-Calvache, Ricardo y Palomares-Linares, Isabel (2015). «Place Attachment and the decision to stay in the neighbourhood». Population Space and Place [en línea], 23 (2). $<$ http://dx.doi.org/10.1002/psp.2001>.

Clark, William A.V. y LisowsKI, William (2017). «Decisions to move and decisions to stay: Life course events and mobility outcomes». Housing Studies [en línea], 32 (5), 547-565. <http://dx.doi.org/10.1080/02673037.2016.1210100>.

Cooke, Thomas. J. (2011). "It is not Just the Economy: Declining Migration and the Rise of Secular Rootedness». Population, Space and Place [en línea], 17, 193-203. <http://dx.doi.org/10.1002/psp.670>.

COUlTER, Rory y VAN HAM, Maarten (2013). «Following people through time: An analysis of individual residential mobility biographies». Housing Studies [en línea], 28 (7), 1037-1055. <http://dx.doi.org/10.1080/02673037.2013.783903>.

Coulter, Rory; van Ham, Maarten y Findlay, Adam M. (2016). «Re-thinking residential mobility: Linking lives through time and space». Progress in Human Geography [en línea], 40 (3), 352-374. <http://dx.doi.org/10.1177/0309132515575417>.

Courgeau, Daniel (1988). «Méthodes de mesure de la mobilité spatiale: Migrations internes, mobilité temporaire et navettes». Population [en línea], 43 (4/5), 877-880. <http://dx.doi.org/10.2307/1533496>.

Courgeau, Daniel y Lelièvre, Eva (1992). «Interrelations between first homeownership, constitution of the family, and professional occupation in France». En: Trussell, James; HanKinson, Richard y Tilton, Judith (eds.). Demographic Applications of Event History Analysis. Oxford: Clarendon Press, 120-140. 
Crowder, Kyle y South, Scott J. (2005). «Race, class, and changing patterns of migration between poor and nonpoor neighborhoods». American Journal of Sociology [en línea], 110, 1715-1763. <http://dx.doi.org/10.1086/428686>.

DAVANZO, Julie (1981). «Repeat migration, information costs, and location-specific capital». Population and Environment [en línea], 4 (1), 45-73. <http://dx.doi.org/10.1007/BF01362575>.

DAWKINS, Cassey J. (2006). «Are social networks the ties that bind families to neighborhoods?». Housing Studies [en línea], 21, 867-888. <http://dx.doi.org/10.1080/02673030600917776>.

Dieleman, Frans M. (2001). "Modelling Residential Mobility: A Review of Recent Trends in Research». Journal of Housing and the Built Environment [en línea], 16, 249-265. <http://dx.doi.org/10.1023/A:1012515709292>.

Dieleman, Frans M. y Everaers, Pieter C.J. (1994). "From renting to owning: Life course and housing market circumstances». Housing Studies [en línea], 9 (1), 11-25. <http://dx.doi.org/10.1080/02673039408720772>.

Elder, Glen H. (1975). "Age differentiation and the life course». Annual Review of Sociology [en línea], 1, 165-190. <http://dx.doi.org/10.1146/annurev.so.01.080175.001121>.

- (1985). Life course dynamics: Trajectories and transitions, 1968-1980. Ithaca: Cornell University Press.

Fischer, Peter A. y Malmberg, Gunnar (2001). «Settled people don’t move: On life course and (im-)mobility in Sweden». International Journal of Population Geography [en línea], 7, 357-371. <http://dx.doi.org/10.1002/ijpg.230>.

Fors, Stephan y LENNARTSSON, Carin (2008). "Social mobility, geographical proximity and intergenerational family contact in Sweden». Ageing \& Society [en línea], 28, 253-270. <http://dx.doi.org/10.1017/S0144686X07006617>.

GoOdman, John L. (1976). "Housing consumption disequilibrium and local residential mobility». Environment and Planning $A$ [en línea], 8, 855-874. <http://dx.doi.org/10.1068/a080855>.

Helderman, Amanda; Mulder, Clara H. y van Ham, Maarten (2004). «The changing effect of home ownership on residential mobility in the Netherlands, 198098». Housing Studies [en línea], 19, 601-616. <http://dx.doi.org/10.1080/0267303042000221981>.

Hiscock, Rosemary; Kearns, Ade; Macintyre, Sally y Ellaway, Anne (2001). «Ontological security and psycho-social benefits from the home: Qualitative evidence on issues of tenure». Housing, Theory and Society [en línea], 18, 50-66. <http://dx.doi.org/10.1080/14036090120617>.

HJäLM, Anna (2014). "The "Stayers": Dynamics of Lifelong Sedentary Behaviour in an Urban Context». Population, Space and Place [en línea], 20 (6), 569-580. <http://dx.doi.org/10.1002/psp.1796>.

Holdsworth, Clare e Irazoqui Solda, Mariana (2002). «First Housing Moves in Spain: An Analysis of Leaving Home and First Housing Acquisition». European Journal of Population [en línea], 18 (1), 1-19. <http://dx.doi.org/10.1023/A:1013831818482>. 
Kan, Kamhon (1999). "Expected and unexpected residential mobility». Journal of Urban Economics [en línea], 45 (1), 72-96. <http://dx.doi.org/10.1006/juec.1998.2082>.

- (2007). «Residential mobility and social capital». Journal of Urban Economics [en línea], 61 (3), 436-457. <http://dx.doi.org/10.1016/j.jue.2006.07.005>.

KemenY, Jim (1991). Housing and social theory [en línea]. Londres: Routledge. <https://doi.org/10.4324/9780203413562>.

KENDIG, Hal L. (1984). «Housing careers, life cycle and residential mobility: Implications for the housing market». Urban Studies [en línea], 21, 271-283. <http://dx.doi.org/10.1080/00420988420080541>.

KlEINHANS, Reinout (2009). "Does social capital affect residents' propensity to move from restructured neighbourhoods?». Housing Studies [en línea], 24 (5), 629-651. <http://dx.doi.org/10.1080/02673030903085784>.

LEAL, Jesús (coord.) (2010). La política de vivienda en España. Madrid: Editorial Pablo Iglesias.

LeE, Barret A.; Oropesa, R.S. y Kanan, James W. (1994). «Neighborhood context and residential mobility». Demography [en línea], 31 (2), 249-270. <https://doi.org/10.2307/2061885>.

Leslie GERALD, R. y RichaRDSON, Arthur H. (1961). «Life-cycle, career pattern, and the decision to move». American Sociological Review [en línea], 26 (6), 894-902. $<$ https://doi.org/10.2307/2090574>.

LONG, Larry (1991). "Residential mobility differences among developed countries». International Regional Science Review [en línea], 14 (2), 133-147. <http://dx.doi.org/10.1177/016001769101400202>.

Meeus, Bruno y De Decker, Pascal (2015). «Staying Put!: A Housing Pathway Analysis of Residential Stability in Belgium». Housing Studies [en línea], 30 (7), 1116-1134. <http://dx.doi.org/10.1080/02673037.2015.1008424>.

Michielin, Francesca y Mulder, Clara H. (2008). «Family events and the residential mobility of couples». Environment and Planning $A$ [en línea], 40, 2770-2790. <http://dx.doi.org/10.1068/a39374>.

MÓDENES, Juan Antonio y CABRÉ, Anna (2002). Flujos espaciales e itinerarios biográficos: La movilidad residencial en el área de Barcelona. Barcelona: Universitat Autònoma de Barcelona.

Módenes, Juan Antonio y López-Colás, Julián (2014). «Recent Demographic Change and Housing in Spain: Towards a New Housing System?». Revista Española de Investigaciones Sociológicas [en línea], 148, 103-134. <http://dx.doi.org/10.5477/cis/reis.148.103>.

MOrrison, Philip y Clark, William A.V. (2016). "Loss aversion and duration of residence». Demographic Research [en línea], 35-36, 1079-1100. <http://dx.doi.org/10.4054/DemRes.2016.35.36>.

Mulder, Clara H. (2006). «Home-ownership and family formation». Journal of Housing and the Built Environment [en línea], 21 (3), 281-298. $<$ http://dx.doi.org/10.1007/s10901-006-9050-9>.

Mulder, Clara H. y CoOKe, Thomas J. (2009). "Family ties and residential locations». Population, Space and Place [en línea], 15, 299-304. <http://dx.doi.org/10.1002/psp.556>. 
Mulder, Clara H. y Hoolmeijer, Pieter (1999). "Residential relocations in the life course». En: Wissen, L.J.G. van y DyKSTRA, P.A. (eds.). Population Studies: An Interdisciplinary Focus [en línea], 159-186. Nueva York: Plenum Press. <http://dx.doi.org/10.1007/978-94-011-4389-9_6>.

Mulder, Clara H. y Manting, Dorien (1994). "Strategies of nest-leavers: "Settling down" versus flexibility». European Sociological Review [en línea], 10 (2), $155-172$. <http://dx.doi.org/10.1093/oxfordjournals.esr.a036327>.

Nivalainen, Satu (2004). "Determinants of family migration: Short moves vs. long moves». Journal of Population Economics [en línea], 17 (1), 157-175. <http://dx.doi.org/10.1007/s00148-003-0131-8>.

OISHI, Shigehiro y TALHELM, Thomas (2012). «Residential mobility what psychological research reveals». Current Directions in Psychological Science [en línea], 21 (6), 425-430. <http://dx.doi.org/10.1177/0963721412460675>.

PABlos, Juan Carlos de y SusinO, Joaquín (2010). «Vida urbana: Entre la desigualdad social y los espacios del habitar». Anduli, 9, 119-142.

Palomares-Linares, Isabel y VAn Ham, Maarten (2016). "Understanding the Effects of Homeownership and Regional Unemployment Levels on Migration during the Economic Crisis in Spain». IZA Discussion Papers, 10232.

Quigley, J.M. (2002). «Transactions Costs and Housing Markets». En: O’Sullivan, Tony y GibB, Kenneth (eds.). Housing Economics and Public Policy [en línea]. Oxford: Blackwell Science Ltd. <http://dx.doi.org/10.1002/9780470690680.ch4>.

RABE, Birgitta y TAYLOR, Mark (2010). "Residential mobility, quality of neighbourhood and life-course events». Journal of the Royal Statistical Society [en línea], 173 (3), 531-555. <http://dx.doi.org/10.1111/j.1467-985X.2009.00626.x>.

Rossi, Peter H. (1955). Why Families Move: A Study in the Social Psychology of Urban Residential Mobility. Glencoe: Free Press.

SABAGH, Georges; ArSDOL, Maurice D. van y BuTlER, Edgar W. (1969). «Some Determinants of Intrametropolitan Residential Mobility: Conceptual Considerations». Social Forces [en línea], 48 (1), 88-98. <http://dx.doi.org/10.1093/sf/48.1.88>.

Salinas-Rodríguez, Aarón; Manrique-Espinoza, Betty y Sosa-Rubí, Sandra G. (2009). "Análisis estadístico para datos de conteo: Aplicaciones para el uso de los servicios de salud». Salud Pública de México [en línea], 51 (5), 397-406. <http://dx.doi.org/10.1590/S0036-36342009000500007>.

SMiTS, Annika (2010). "Moving close to parents and adult children in the Netherlands: The influence of support needs». Demographic Research [en línea], 22, 985-1014. <http://dx.doi.org/10.4054/DemRes.2010.22.31>.

SPEARE, Alden (1970). "Home ownership, life cycle stage, and residential mobility». Demography [en línea], 7 (4), 449-458. <https://doi.org/10.2307/2060237>.

STOVEL, Katherine y Bolan, Mark (2004). «Residential trajectories: Using optimal alignment to reveal the structure of residential mobility». Sociological Methods \& Research [en línea], 32 (4), 559-598. <http://dx.doi.org/10.1177/0049124103262683>. 
Susino, Joaquín y Duque-Calvache, Ricardo (2013). «Veinte años de suburbanización en España (1981-2001): El perfil de sus protagonistas». Documents d'Anàlisi Geogràfica [en línea], 59 (2), 265-290. $<$ http://dx.doi.org/10.5565/rev/dag.31>.

Susino, Joaquín y PALOMARES-LinARES, Isabel (2013). «La movilidad residencial en el área metropolitana de Granada». En: CAMACHO BALlesta, Juan Antonio y Jiménez Olivencia, Yolanda (eds.). Desarrollo Regional Sostenible en tiempos de crisis. Granada: Universidad de Granada, 345-363.

Thomas, Michael J.; STILlwell, John C.H. y Gould, Myles (2015). «Modelling Mover/Stayer Characteristics across the Life Course Using a Large Commercial Sample». Population Space and Place [en línea], 22, 584-598. <http://dx.doi.org/10.1002/psp.1943>.

- (2016). «Modelling the duration of residence and plans for future residential relocation: A multilevel analysis». Transactions of the Institute of British Geographers [en línea], 41 (3), 297-312. <http://dx.doi.org/10.1111/tran.12123>.

van Ham, Maarten; Clark, William, A.V. (2009). "Neighbourhood mobility in context: Household moves and changing neighbourhoods in the Netherlands». Environment and Planning $A$ [en línea], 41, 1442-1459. $<$ http://dx.doi.org/10.1068/a4182>.

van Ham, Maarten; Hedman, Lina; Manley, David; Coulter, Rory y Östh, John (2014). "Intergenerational transmission of neighbourhood poverty: An analysis of neighbourhood histories of individuals». Transactions of the Institute of British Geographers [en línea], 39, 402-417. <http://dx.doi.org/10.1111/tran.12040>.

WARNES, Alan M. (1986). "The residential mobility histories of parents and children, and relationships to present proximity and social integration». Environment and Planning A [en línea], 18 (12), 1581-1594. $<$ http://dx.doi.org/10.1068/a181581>.

WeINBERG, Daniel H.; Friedman, Joseph y MaYO, Stephen K. (1981). «Intraurban residential mobility: The role of transaction costs, market imperfections, and household disequilibrium». Journal of Urban Economics [en línea], 9, 332-348. <http://dx.doi.org/10.1016/0094-1190(81)90031-0>.

Winstanley, Ann; Thorns, David C. y Perkins, Harvey. C. (2002). «Moving house, creating home: Exploring residential mobility». Housing Studies [en línea], 17 (6), 813-832. <http://dx.doi.org/10.1080/02673030216000>. 


\section{Anexos}

Tabla A1. Probabilidades predichas para cada categoría por el modelo Hurdle. Variable (in)movilidad residencial

\begin{tabular}{|c|c|c|c|c|}
\hline & 0 cambios & 1 cambio & 2 cambios & 3 cambios \\
\hline Prob. predicha media & 0,5599 & 0,3463 & 0,0715 & 0,0161 \\
\hline \multicolumn{5}{|l|}{$\overline{\text { Edad }}$} \\
\hline $18-29$ & 0,3338 & 0,4827 & 0,1480 & 0,0302 \\
\hline $30-39$ & 0,4234 & 0,4565 & 0,1028 & 0,0154 \\
\hline $40-49$ & 0,5183 & 0,4064 & 0,0673 & 0,0074 \\
\hline $50-59$ & 0,6119 & 0,3428 & 0,0417 & 0,0034 \\
\hline $60-69$ & 0,6979 & 0,2759 & 0,0247 & 0,0015 \\
\hline $70-79$ & 0,7720 & 0,2134 & 0,0140 & 0,0006 \\
\hline 80 o más & 0,8323 & 0,1598 & 0,0077 & 0,0002 \\
\hline \multicolumn{5}{|c|}{ Posición socioeconómica (ref.: Trab. admin.) } \\
\hline Empresarios o profesionales & 0,5969 & 0,3441 & 0,0531 & 0,0055 \\
\hline Trabajadores de los servicios & 0,5779 & 0,3552 & 0,0596 & 0,0067 \\
\hline Trabajadores manuales & 0,5933 & 0,3604 & 0,0427 & 0,0034 \\
\hline Otros y no clasificables & 0,6380 & 0,3113 & 0,0458 & 0,0045 \\
\hline \multicolumn{5}{|l|}{ Estructura de hogar (ref.: Parejas) } \\
\hline Unipersonal & 0,4250 & 0,4656 & 0,0950 & 0,0129 \\
\hline Familias & 0,5766 & 0,3662 & 0,0519 & 0,0049 \\
\hline Otros hogares & 0,7422 & 0,2285 & 0,0270 & 0,0021 \\
\hline \multicolumn{5}{|l|}{$\begin{array}{l}\text { Tenencia de vivienda } \\
\text { (ref.: Propietarios con pagos pendientes) }\end{array}$} \\
\hline Propietarios sin cargas & 0,8073 & 0,1761 & 0,0156 & 0,0009 \\
\hline Alquiler & 0,2655 & 0,5199 & 0,1703 & 0,0372 \\
\hline Otras formas & 0,6901 & 0,2779 & 0,0298 & 0,0021 \\
\hline \multicolumn{5}{|l|}{$\begin{array}{l}\text { Tipo de sección censal } \\
\text { (ref.: Secciones clases medias) }\end{array}$} \\
\hline Secciones acomodadas & 0,6475 & 0,3166 & 0,0334 & 0,0024 \\
\hline Secciones de clases medias mixtas & 0,6086 & 0,3366 & 0,0495 & 0,0049 \\
\hline Secciones populares & 0,5431 & 0,4049 & 0,0479 & 0,0038 \\
\hline Secciones deprimidas & 0,5970 & 0,3582 & 0,0414 & 0,0032 \\
\hline Nuevas secciones & 0,5045 & 0,4244 & 0,0642 & 0,0065 \\
\hline
\end{tabular}

Fuente: Ayuntamiento de Granada. Encuesta sobre vivienda y población metropolitana, 2008. 
Tabla A2. Probabilidades predichas para cada categoría por el modelo Hurdle. Variable (in) movilidad espacial

\begin{tabular}{|c|c|c|c|c|}
\hline & 0 cambios & 1 cambio & 2 cambios & 3 cambios \\
\hline Prob. predicha media & 0,3492 & 0,5181 & 0,1012 & 0,0228 \\
\hline \multicolumn{5}{|l|}{ Edad } \\
\hline $18-29$ & 0,3626 & 0,4549 & 0,1457 & 0,0311 \\
\hline $30-39$ & 0,3496 & 0,5126 & 0,1176 & 0,0180 \\
\hline $40-49$ & 0,3369 & 0,5602 & 0,0920 & 0,0101 \\
\hline $50-59$ & 0,3243 & 0,5993 & 0,0705 & 0,0055 \\
\hline $60-69$ & 0,3121 & 0,6316 & 0,0532 & 0,0030 \\
\hline $70-79$ & 0,3000 & 0,6586 & 0,0397 & 0,0016 \\
\hline 80 o más & 0,2883 & 0,6814 & 0,0295 & 0,0008 \\
\hline \multicolumn{5}{|c|}{ Posición socioeconómica (ref.: Trab. admin.) } \\
\hline Empresarios o profesionales & 0,2866 & 0,5853 & 0,1122 & 0,0143 \\
\hline Trabajadores de los servicios & 0,2868 & 0,5975 & 0,1028 & 0,0118 \\
\hline Trabajadores manuales & 0,4234 & 0,4962 & 0,0727 & 0,0071 \\
\hline Otros y no clasificables & 0,2984 & 0,5800 & 0,1071 & 0,0132 \\
\hline \multicolumn{5}{|l|}{ Estructura de hogar (ref.: Parejas) } \\
\hline Unipersonal & 0,2688 & 0,5649 & 0,1400 & 0,0231 \\
\hline Familias & 0,3379 & 0,5632 & 0,0887 & 0,0093 \\
\hline Otros hogares & 0,3952 & 0,5256 & 0,0721 & 0,0066 \\
\hline \multicolumn{5}{|l|}{$\begin{array}{l}\text { Tenencia de vivienda } \\
\text { (ref.: Propietarios con pagos pendientes) }\end{array}$} \\
\hline Propietarios sin cargas & 0,3702 & 0,5855 & 0,0421 & 0,0020 \\
\hline Alquiler & 0,3940 & 0,4191 & 0,1461 & 0,0340 \\
\hline Otras formas & 0,4242 & 0,5082 & 0,0622 & 0,0051 \\
\hline \multicolumn{5}{|l|}{$\begin{array}{l}\text { Tipo de sección censal } \\
\text { (ref.: Secciones clases medias) }\end{array}$} \\
\hline Secciones acomodadas & 0,3008 & 0,6192 & 0,0737 & 0,0059 \\
\hline Secciones de clases medias mixtas & 0,3223 & 0,5545 & 0,1077 & 0,0140 \\
\hline Secciones populares & 0,3587 & 0,5723 & 0,0640 & 0,0048 \\
\hline Secciones deprimidas & 0,3810 & 0,5472 & 0,0662 & 0,0053 \\
\hline Nuevas secciones & 0,2976 & 0,5652 & 0,1187 & 0,0166 \\
\hline
\end{tabular}

Fuente: Ayuntamiento de Granada. Encuesta sobre vivienda y población metropolitana, 2008. 